\title{
A STRAIGHTENING LAW FOR THE DRINFEL'D LAGRANGIAN GRASSMANNIAN
}

\author{
A Dissertation \\ by \\ JAMES VINCENT RUFFO
}

\begin{abstract}
Submitted to the Office of Graduate Studies of Texas A\&M University in partial fulfillment of the requirements for the degree of DOCTOR OF PHILOSOPHY
\end{abstract}

August 2007

Major Subject: Mathematics 


\title{
A STRAIGHTENING LAW FOR THE \\ DRINFEL'D LAGRANGIAN GRASSMANNIAN
}

\author{
A Dissertation \\ by \\ JAMES VINCENT RUFFO
}

\begin{abstract}
Submitted to the Office of Graduate Studies of Texas A\&M University in partial fulfillment of the requirements for the degree of

DOCTOR OF PHILOSOPHY
\end{abstract}

Approved by:

Chair of Committee, Frank Sottile

Committee Members, John Keyser

J. Maurice Rojas

Henry Schenck

Head of Department, Al Boggess

August 2007

Major Subject: Mathematics 


\author{
ABSTRACT \\ A Straightening Law for the \\ Drinfel'd Lagrangian Grassmannian. (August 2007) \\ James Vincent Ruffo, B.A.;B.S., University of Rochester; \\ M.A., University of Massachusetts-Amherst \\ Chair of Advisory Committee: Dr. Frank Sottile
}

The Drinfel'd Lagrangian Grassmannian compactifies the space of algebraic maps of fixed degree from the projective line into the Lagrangian Grassmannian. It has a natural projective embedding arising from the highest weight embedding of the ordinary Lagrangian Grassmannian, and one may study its defining ideal in this embedding. The Drinfel'd Lagrangian Grassmannian is singular. However, a concrete description of generators for the defining ideal of the Schubert subvarieties of the Drinfel'd Lagrangian Grassmannian would imply that the singularities are modest.

I prove that the defining ideal of any Schubert subvariety is generated by polynomials which give a straightening law on an ordered set. Using this fact, I show that any such subvariety is Cohen-Macaulay and Koszul. These results represent a partial extension of standard monomial theory to the Drinfel'd Lagrangian Grassmannian. 


\section{ACKNOWLEDGMENTS}

I thank my advisor Frank Sottile for introducing me to this topic, and for his sup-

port and guidance. I also thank Dimitrije Kostić, J.M. Landsberg, Mauricio Velasco and Alexander Woo for helpful conversations and suggestions. 


\section{TABLE OF CONTENTS}

CHAPTER

INTRODUCTION . . . . . . . . . . . . . . . . . . . 1

II

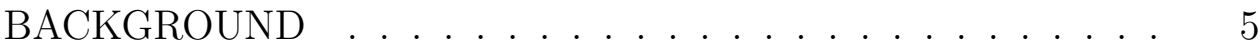

A. Basic theory . . . . . . . . . . . . . 5

1. Algebraic geometry . . . . . . . . . . . 5

2. Representation theory . . . . . . . . . . . 10

B. Flag varieties . . . . . . . . . . . . . . . 16

1. The Lagrangian Grassmannian . . . . . . . . . . . . 16

C. Spaces of algebraic maps . . . . . . . . . . . . . . . 26

D. Algebras with straightening laws . . . . . . . . . . . 28

1. Hilbert series of an algebra with straightening law . . 31

2. The doset of admissible pairs . . . . . . . . . 36

III RESULTS ...................... 40

A. Algebras with straightening law . . . . . . . . . . 40

B. Drinfel'd flag varieties . . . . . . . . . . . . . . . 42

1. A basis for $S_{d} \mathbb{C}^{2} \otimes \mathrm{L}\left(\omega_{n}\right)^{*} \ldots . . . . . . . . .442$

2. Proof and consequences of the straightening law . . . 50

3. Representation-theoretic interpretation . . . . . . 56

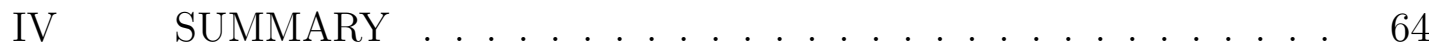

REFERENCES . . . . . . . . . . . . . . . . . . . 66

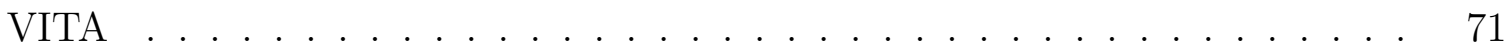




\section{LIST OF FIGURES}

FIGURE

1 The partition $(3,3,1)$ associated to $\overline{4} \overline{2} 23 . \quad \ldots \ldots$. . . . . . . . . . 20

2 The paths associated to $\overline{4} \overline{2} 13$ and $\overline{3} \overline{1} 24$ in $\mathcal{P}_{4}$. . . . . . . . . . . . . . 23

$3 \quad$ Elements of $\pi_{n}^{-1}(\overline{4} \overline{2} 13, \overline{3} \overline{1} 24) \ldots \ldots \ldots \ldots$. . . . . . . . . . . . . . . .

$4 \quad$ The set $\operatorname{Ch}(\mathcal{D})$ of chains in $\mathcal{D} \ldots \ldots \ldots$. . . . . . . . . . . 32

5 The doset of Example 2.50 . . . . . . . . . . . . . . . 36

$6 \quad$ The doset $\mathcal{D}_{2,4} \ldots \ldots \ldots \ldots$. . . . . . . . . . . . . . . . . .

$7 \quad$ The subset of $S_{2,4}$ associated to $\overline{4} \overline{2} 13^{(1)} \in \mathcal{P}_{2,4} \ldots \ldots$. . . . . . . . . . 39

8 The two cases in the proof of fourth condition in Definition 2.45. . . 52

$9 \quad$ The module $S^{2}\left(S^{d} \mathbb{C}^{2} \otimes \mathrm{L}(\omega)\right)$ decomposes into factors corresponding to the colored boxes. . . . . . . . . . . . . . . . 59

$10 \quad$ A Young scheme on $\lambda / \mu(-1) \ldots \ldots \ldots$. . . . . . . . . . 61

$11 \quad$ A Young scheme on $\lambda / \mu \ldots \ldots \ldots$. . . . . . . . . . . 61 


\section{CHAPTER I}

\section{INTRODUCTION}

Enumerative geometry concerns problems of counting geometric objects incident upon other objects in a prescribed way. In the second half of the $19^{\text {th }}$ century, many remarkable numbers in enumerative geometry were computed - for example, in 1864 Chasles proved that there are 3264 plane conics tangent to 5 fixed conics [20].

The Schubert calculus is concerned with an important subset of enumerative geometry. It provides a framework for counting linear subspaces of a vector space satisfying conditions imposed by other linear subspaces. We call a $k$-dimensional linear subspace a $k$-plane, or if $k=1$, a line. Note that we require that these objects pass through the origin. The smallest non-trivial example of a problem in the Schubert calculus is the following.

Question 1.1. Given four general fixed 2-planes in $\mathbb{C}^{4}$, how many 2-planes meet all of them?

It turns out that the answer is 2. The use of the term "general" is in the technical sense of algebraic geometry. We think of the four 2-planes as being fixed in space, and the problem is to count the number of ways to place a 2-plane in some position so that it intersects each of the four 2-planes non-trivially. We thus refer to the objects imposing the conditions as fixed.

More generally, one may ask:

Question 1.2. How many $k$-planes in $n$-dimensional space meet $k(n-k)$ fixed general $n-k$-planes non-trivially?

This dissertation follows the style of Representation Theory. 
The answer, first computed by Schubert [37], is

$$
\frac{1 ! 2 ! \cdots k !(k(n-k)) !}{(n-k) !(n-k+1) ! \cdots n !} \text {. }
$$

The Schubert calculus deals not only with linear subspaces, but with nested chains $E_{\bullet}:=\left(E_{a_{1}} \subset E_{a_{2}} \subset \cdots \subset E_{a_{s}} \subset \mathbb{C}^{n}\right)$ with $E_{a_{i}}$ an $a_{i}$-plane in $\mathbb{C}^{n}$, for each $i=1, \ldots, s$. We say that $E_{\bullet}$ is a flag of type $\left(a_{1}, a_{2}, \ldots, a_{s}\right)$ in $\mathbb{C}^{n}$. An example of a problem involving flags is answered by the following proposition.

Proposition 1.3. There are 2 flags $\left(E_{2} \subset E_{3}\right)$ of type $(2,3)$ in 4-dimensional space $\mathbb{C}^{4}$ such that $E_{2}$ meets 3 general fixed 2-planes and $E_{3}$ contains 2 fixed lines.

In the modern setting, these problems are solved by introducing a parameter space for the objects to be counted, called a flag variety. This is a smooth projective variety, and it has subvarieties (called Schubert varieties) which parametrize flags satisfying incidence conditions with respect to a fixed flag. (see Chapter II for background material). The problem is thus reduced to the problem of computing the number of points in an intersection of Schubert varieties. These points are the set of solutions to a system of polynomial equations determined by the Schubert varieties.

Flag varieties are the compact homogeneous spaces for the action of a complex semisimple algebraic group $G$. All of the examples above involve flag varieties associated to a special linear group. For us, the symplectic group $\operatorname{Sp}_{2 n}(\mathbb{C})$ will figure prominently.

Example 1.4. Let $\Omega$ be a non-degenerate alternating bilinear form on the vector space $\mathbb{C}^{4}$. The Lagrangian Grassmannian $X:=\mathrm{LG}(2)$ is the set of all 2-planes $E \subset \mathbb{C}^{4}$ such that $\Omega$ is identically zero on $E$. Such subspaces are called isotropic.

The symplectic group $G:=\mathrm{Sp}_{4} \mathbb{C}$ is the group of invertible linear transformations $T: \mathbb{C}^{4} \rightarrow \mathbb{C}^{4}$ such that $\Omega(T(v), T(w))=\Omega(v, w)$ for all $v, w \in \mathbb{C}^{4}$. The group $G$ acts 
transitively on $X$. Pick a point $x \in X$, and let $P \subset G$ be its stabilizer. There is a bijection $X \cong G / P$. In the literature the terms "flag variety" and $G / P$ are used interchangeably.

One may pose enumerative questions on $X$, for example:

Question 1.5. How many isotropic 2-planes $x \in X$ meet 3 general isotropic 2-planes?

Again, the answer is 2 .

Recently there has been much interest in enumerative problems involving algebraic maps from a complete curve $C$ to a flag variety. These problems involve conditions that the image of such a map pass through a Schubert variety at some point $c \in C$, and can be studied using the quantum cohomology ring of the flag variety [14].

These problems can be posed as intersections on an auxiliary space parametrizing such algebraic maps. This space has applications to mathematical physics, linear systems theory, geometric representation theory, and the geometric Langlands correspondence, as well as quantum cohomology (see $[2,39,40]$ for details). It is (almost) never compact, so various compactifications have been introduced to help understand its geometry. Among these are Kontsevich's space of stable maps [14, 21], the quot scheme (or space of quasiflags) $[6,32,42]$, and, at least when $C$ is the projective line $\mathbb{P}^{1}$, the Drinfel'd compactification (or space of quasimaps) [2, 22]. This latter space is defined concretely as a projective variety, and it is amenable to direct study via its defining equations. In particular, one may apply some of the ideas of standard monomial theory.

Inspired by the work of Hodge [19] on the Grassmannian, Lakshmibai, Musili, Seshadri, and others (see $[23,35]$ and references therein) developed standard monomial theory to study the flag varieties $G / Q$, where $G$ is a semisimple algebraic group 
and $Q \subset G$ is a parabolic subgroup. These spaces have a decomposition into Schubert cells, whose closures (the Schubert varieties) give a basis for cohomology. A consequence of standard monomial theory is that Schubert varieties are normal and Cohen-Macaulay, and one has an explicit description of their singularities and defining ideals.

A key part of standard monomial theory is that any $G / P(P$ a maximal parabolic subgroup) has a projective embedding with coordinates such that the defining ideal of $G / P$ takes the form of a straightening law (Definition 2.45). This idea originates with the work of Hodge on the Grassmannian [19], and was extended to the Lagrangian Grassmannian by DeConcini and Lakshmibai [9]. An algebra with straightening law is a special case of a Hodge algebra [5].

Sottile and Sturmfels have extended standard monomial theory to the Drinfel'd Grassmannian parametrizing algebraic maps from $\mathbb{P}^{1}$ into the Grassmannian [41]. They extend the definition of Schubert varieties to this space, and prove that the homogeneous coordinate ring of any Schubert variety (including the Drinfel'd Grassmannian itself) is an algebra with straightening law on a distributive lattice. This is the key fact that allows one to prove that these Schubert varieties are normal, Cohen-Macaulay, and Koszul, and have rational singularities.

We extend these results to the Drinfel'd Lagrangian Grassmannian, defined in Chapter II, Section C. The necessary background on algebras with straightening law is provided in Chapter II, Section D. Our main results are proved in Chapter III, along with some consequences. 


\section{CHAPTER II}

\section{BACKGROUND}

A. Basic theory

\section{Algebraic geometry}

We review some facts from algebraic geometry, which is the study of the solution sets of systems of polynomial equations. We recall the basic theorems and definitions of the subject, emphasizing those ideas which will be of particular interest to us. See $[11,17,18]$ for more details on the general theory and $[10,16]$ for more on Gröbner bases.

Let $\mathbb{C}\left[x_{1}, \ldots, x_{n}\right]$ denote the ring of polynomials in the variables $x_{1}, \ldots, x_{n}$ with complex coefficients. Let $\mathbb{A}^{n}:=\left\{a=\left(a_{1}, \ldots, a_{n}\right) \mid a_{i} \in \mathbb{C}, i=1, \ldots, n\right\}$ denote $n$-dimensional affine space over $\mathbb{C}$. Associated to a subset $S \subset \mathbb{C}\left[x_{1}, \ldots, x_{n}\right]$ we have the affine variety

$$
\mathcal{V}(S):=\left\{a \in \mathbb{A}^{n} \mid f(a)=0, \text { for all } f \in S\right\}
$$

Conversely, to a subset $X \subset \mathbb{A}^{n}$, we associate the set of polynomials vanishing on $X$

$$
\mathcal{I}(X):=\left\{f \in \mathbb{C}\left[x_{1}, \ldots, x_{n}\right] \mid f(a)=0, \text { for all } a \in X\right\}
$$

These associations form the basis for a correspondence between algebraic and geometric objects. Several refinements must be made to make this correspondence precise.

First, note that for any subset $X \subset \mathbb{A}^{n}, \mathcal{I}(X)$ is an ideal. That is, $0 \in \mathcal{I}(X)$, and $f, g \in \mathcal{I}(X)$ and $b \in \mathbb{C}\left[x_{1}, \ldots, x_{n}\right]$ imply that $f+b g \in \mathcal{I}(X)$. Hence we may restrict attention to ideals of $\mathbb{C}\left[x_{1}, \ldots, x_{n}\right]$. Hilbert's Basis Theorem [11] implies that every 
such ideal $I$ is finitely generated, i.e., there exist $f_{1}, \ldots, f_{r} \in \mathbb{C}\left[x_{1}, \ldots, x_{n}\right]$ such that

$$
I=\left\{g_{1} f_{1}+\cdots+g_{r} f_{r} \mid g_{1}, \ldots, g_{r} \in \mathbb{C}\left[x_{1}, \ldots, x_{n}\right]\right\}
$$

We write $I=\left\langle f_{1}, \ldots, f_{r}\right\rangle$ in this situation.

For a given variety $X \subset \mathbb{A}^{n}$, there are many ideals $I$ such that $\mathcal{V}(I)=X$, and $\mathcal{I}(X)$ contains all of them. Ideals of the form $\mathcal{I}(X)$ can be characterized purely in terms of algebra. The radical of $I$, denoted $\sqrt{I}$, is the ideal of all $f \in \mathbb{C}\left[x_{1}, \ldots, x_{n}\right]$ such that $f^{N} \in I$, for some $N \in \mathbb{N}$. $I$ is a radical ideal if $I=\sqrt{I}$.

Theorem 2.1 (Hilbert's Nullstellesatz). Let $I \subset \mathbb{C}\left[x_{1}, \ldots, x_{n}\right]$ be an ideal. Then

$$
\mathcal{I}(\mathcal{V}(I))=\sqrt{I}
$$

We can now state one version of the algebro-geometric dictionary:

Proposition 2.2. The maps $X \mapsto \mathcal{I}(X)$ and $I \mapsto \mathcal{V}(I)$ give an inclusion reversing bijection between radical ideals in the ring $\mathbb{C}\left[x_{1}, \ldots, x_{n}\right]$ and affine varieties contained in $\mathbb{A}^{n}$.

In a similar fashion, one may consider subvarieties of projective space. Recall that $n$-dimensional projective space $\mathbb{P}^{n}$ is the set of all 1-dimensional linear subspaces of the $n+1$-dimensional vector space $\mathbb{C}^{n+1}$. Let $x_{0}, \ldots, x_{n}$ be coordinate functions on $\mathbb{C}^{n+1}$ (that is, a basis for the dual vector space $\mathbb{C}^{n+1^{*}}$ ). These are homogeneous coordinates on $\mathbb{P}^{n}$. A projective variety is the set of zeroes in $\mathbb{P}^{n}$ of some set of polynomials in $\mathbb{C}\left[x_{0}, \ldots, x_{n}\right]$. A polynomial in $\mathbb{C}\left[x_{0}, \ldots, x_{n}\right]$ can be written as $f=\sum_{i=1}^{N} f_{i}$, where each $f_{i}$ is a homogeneous polynomial of degree $i$; the polynomial $f$ vanishes on a point of $\mathbb{P}^{n}$ if and only if each homogeneous component $f_{i}$ does so. Hence the defining ideal of a projective variety is homogeneous, in that it is generated by a set of homogeneous polynomials. 
Note that the irrelevant ideal $\left\langle x_{0}, \ldots, x_{n}\right\rangle$ defines the empty set in $\mathbb{P}^{n}$, as does any homogeneous ideal containing some power $\left\langle x_{0}, \ldots, x_{n}\right\rangle^{m}(m \in \mathbb{N})$ of this ideal. Moreover, a polynomial $f \in \mathbb{C}\left[x_{0}, \ldots, x_{n}\right]$ vanishes on a given projective variety if and only if for some $N$, all of the polynomials $x_{0}^{N} f, \ldots, x_{n}^{N} f$ vanish. This motivates the following definition.

Definition 2.3. The saturation of an ideal $I \subset \mathbb{C}\left[x_{0}, \ldots, x_{n}\right]$ is the ideal

sat $I=\left\{f \in \mathbb{C}\left[x_{0}, \ldots, x_{n}\right] \mid x_{i}^{N} f \subset I\right.$ for all $i=0, \ldots, n$ and some $\left.N \in \mathbb{N}\right\}$.

The ideal $I$ is saturated if sat $I=I$.

We again write $\mathcal{V}(I)$ for the zero set in $\mathbb{P}^{n}$ of a homogeneous ideal and $\mathcal{I}(X)$ for the defining ideal of a projective variety. One has the following homogeneous version of the algebro-geometric dictionary.

Proposition 2.4. The maps $X \mapsto \mathcal{I}(X)$ and $I \mapsto \mathcal{V}(I)$ give an inclusion reversing bijection between saturated radical homogeneous ideals in the ring $\mathbb{C}\left[x_{0}, \ldots, x_{n}\right]$ and projective varieties in $\mathbb{P}^{n}$.

Gröbner bases have driven a resurgence of computational methods in algebraic geometry in the past two decades. We recall the basics.

We write $x^{a}$ for the monomial $x_{1}^{a_{1}} \cdots x_{n}^{a_{n}} \in \mathbb{C}\left[x_{1}, \ldots, x_{n}\right]$. Let $\operatorname{deg} x^{a}:=\sum_{i=1}^{n} a_{i}$ be the total degree of $x^{a}$.

A term order $<$ is a total ordering on the set of monomials in $\mathbb{C}\left[x_{1}, \ldots, x_{n}\right]$ such that 1 is the unique minimal element of $\mathbb{C}\left[x_{1}, \ldots, x_{n}\right]$, and such that for any monomials $m_{1}, m_{2}$, and $m$ in $\mathbb{C}\left[x_{1}, \ldots, x_{n}\right], m_{1}<m_{2}$ implies that $m m_{1}<m m_{2}$.

Consider the linear ordering of the variables given by $x_{1}>x_{2}>\ldots>x_{n}$. Some of the most frequently used term orders are the following: 
1. $x^{a} \leq x^{b}$ if and only if the first nonzero entry of $b-a$ is positive. This defines the lexicographic term order on $\mathbb{C}\left[x_{1}, \ldots, x_{n}\right]$.

2. $x^{a} \leq x^{b}$ if and only if $\operatorname{deg} x^{a}<\operatorname{deg} x^{b}$, or $\operatorname{deg} x^{a}=\operatorname{deg} x^{b}$ and the first nonzero entry of $b-a$ is positive. This defines the degree lexicographic term order on $\mathbb{C}\left[x_{1}, \ldots, x_{n}\right]$.

3. $x^{a} \leq x^{b}$ if and only if $\operatorname{deg} x^{a}<\operatorname{deg} x^{b}$, or $\operatorname{deg} x^{a}=\operatorname{deg} x^{b}$ and the last nonzero entry of $b-a$ is negative. This defines the degree reverse lexicographic term order on $\mathbb{C}\left[x_{1}, \ldots, x_{n}\right]$.

Given a term order $<$, we define the initial monomial $\operatorname{in}_{<} f$ of $f$ to be the largest monomial (with respect to $<$ ) appearing in $f$. For $I \subset \mathbb{C}\left[x_{1}, \ldots, x_{n}\right]$ an ideal, the initial ideal $\operatorname{in}_{<} I$ is the ideal generated by the set $\left\{\operatorname{in}_{<} f \mid f \in I\right\}$.

Definition 2.5. Let $I \subset \mathbb{C}\left[x_{1}, \ldots, x_{n}\right]$ be an ideal. Then a set $\left\{f_{1}, \ldots, f_{k}\right\} \subset I$ is a Gröbner basis for $I$ if $\operatorname{in}_{<} I$ is generated by $\left\{\operatorname{in}_{<} f_{1}, \ldots, \operatorname{in}_{<} f_{k}\right\}$.

Proposition 2.6. Let $\left\{f_{1}, \ldots, f_{k}\right\} \subset I$ be a Gröbner basis for I. Then I is generated by $\left\{f_{1}, \ldots, f_{k}\right\}$.

Example 2.7. Let $f_{1}:=\underline{x^{2}}-y$ and $f_{2}:=\underline{x^{3}}-z$. Consider the ideal $I:=\left(f_{1}, f_{2}\right) \subset$ $\mathbb{C}[x, y, z]$. Let $<$ denote the lexicographic ordering on $\mathbb{C}[x, y, z]$, so that the initial terms are those that are underlined above. The polynomial

$$
f_{3}:=\underline{x y}-z=x f_{1}-f_{2}
$$

is in $I$, but its initial term $y z^{5}$ does not lie in the ideal generated by $\operatorname{in}_{<} f_{1}$ and $\operatorname{in}_{<} f_{2}$. Thus $\left\{f_{1}, f_{2}\right\}$ is not a Gröbner basis with respect to this term order. One can check that $\left\{f_{1}, f_{2}, f_{3}\right\}$ is a Gröbner basis for $I$. 
Algorithms 2.8 and 2.9 form the computational backbone of the Gröbner basis approach.

Algorithm 2.8 (Division algorithm). Fix a term order $<$ on $\mathbb{C}\left[x_{1}, \ldots, x_{n}\right]$, and a Gröbner basis $G=\left\{g_{1}, \ldots, g_{r}\right\}$.

INPUT: A polynomial $f \in \mathbb{C}\left[x_{1}, \ldots, x_{n}\right]$.

OUTPUT: The remainder upon division of $f$ by $G$.

1. Let $m$ be the largest monomial of $f$ which has not yet been processed.

2. If $\operatorname{in}_{<g_{i}}$ divides $m$ for some $i=1, \ldots, r$ (say, $m^{\prime}$ in $_{<g_{i}}=m$ for some monomial $\left.m^{\prime}\right)$, replace $f$ with $f-m^{\prime} g$. Only monomials which are smaller than $m$ are introduced into $f$.

3. Add $m$ to the list of processed monomials.

4. If no monomial in $f$ is divisible by any $\operatorname{in}_{<} g_{i}$ for $i=1, \ldots, r$, output $f$. Otherwise, return to step 1 .

Starting with a set of polynomials, one obtains a Gröbner basis for the ideal they generate by the following algorithm.

Algorithm 2.9 (Buchberger's algorithm). Fix a term order $<$ on $\mathbb{C}\left[x_{1}, \ldots, x_{n}\right]$.

INPUT: $A$ set $S:=\left\{f_{1}, \ldots, f_{r}\right\} \subset \mathbb{C}\left[x_{1}, \ldots x_{n}\right]$ of polynomials which generate the ideal $I \subset \mathbb{C}\left[x_{1}, \ldots x_{n}\right]$, with $m_{i}:=\operatorname{in}_{<} f_{i}$ the initial term of $f_{i}(i=1, \ldots, r)$. Assume that the coefficient of $m_{i}$ is 1 for each $i=1, \ldots, r$.

OUTPUT: A Gröbner basis for I.

1. For each $i, j \in[r]$, form the $S$-polynomial

$$
S\left(f_{i}, f_{j}\right):=\frac{\operatorname{lcm}\left(m_{i}, m_{j}\right)}{m_{i}} f_{i}-\frac{\operatorname{lcm}\left(m_{i}, m_{j}\right)}{m_{j}} f_{j}
$$

and let $f_{i, j}$ be the reduction of $S\left(f_{i}, f_{j}\right)$ modulo $f_{1}, \ldots, f_{r}$. 
2. If $f_{i, j} \neq 0$, replace $S$ with the set $S \cup\left\{f_{i, j}\right\}$, and return to the first step.

3. If each $S$-polynomial reduces to zero modulo $S$, output $S$.

The $S$-polynomials in Buchberger's algorithm have the following property.

Proposition 2.10. Let $m_{i}=\operatorname{in}_{<} f_{i}$ for $i=1,2$ and polynomials $f_{i} \in \mathbb{C}\left[x_{1}, \ldots, x_{n}\right]$. If $\operatorname{lcm}\left(m_{1}, m_{2}\right)=1$, then $S\left(f_{1}, f_{2}\right)$ reduces to zero modulo $\left\{f_{1}, f_{2}\right\}$.

Proposition 2.10 will be useful for the polynomial systems we study in Chapter III.

\section{Representation theory}

We review the basic facts we need from representation theory. See $[1,13]$ for a more complete account.

Definition 2.11. An (affine) algebraic group is an (affine) algebraic variety $G$ which is a group, such that the maps

$$
\begin{gathered}
\mu: G \times G \rightarrow G \\
\iota: G \longrightarrow G
\end{gathered}
$$

given by $\mu(g, h)=g h$ and $\iota(g)=g^{-1}$ are regular.

Let $G$ be an algebraic group. For any $g \in G$, the map $L_{g}: G \rightarrow G, L_{g}(h):=g h$ is an automorphism of $G$ (as an algebraic variety). In particular, $G$ is smooth, and the differential $d_{h} L_{g}: T_{h} G \rightarrow T_{g h} G$ is an isomorphism for all $h, g \in G$. This allows us to identify the tangent space of $G$ at any point with the tangent space at the identity $\mathfrak{g}:=T_{e} G$. 
For any group $G$, let $[G, G]:=\left\{g h g^{-1} h^{-1} \mid g, h \in G\right\}$ denote its commutator subgroup. The derived series of subgroups is defined inductively by $D_{0}(G):=G$ and $D_{i+1}(G):=\left[D_{i}(G), D_{i}(G)\right]$.

Definition 2.12. A group is solvable if $D_{n}(G)=\{e\}$ for some $n \in \mathbb{Z}_{+}$.

Definition 2.13. Let $G$ be an algebraic group. A Borel subgroup of $G$ is a maximal closed connected solvable subgroup. A torus is a closed connected abelian subgroup.

The subgroup $B$ of upper-triangular matrices in $G=\mathrm{GL}_{n}(\mathbb{C})$ is a Borel subgroup, and the diagonal matrices $T \subset B$ is a maximal torus (in $G$ or in $B$; since an abelian group is solvable, any torus is contained a Borel subgroup). When working with more general groups $G$, it is typically the case that there is a natural embedding of $G$ into $\mathrm{GL}_{n}(\mathbb{C})$ such that the upper-triangular matrices in $G$ form a Borel subgroup of $G$, and similarly for a maximal torus.

Definition 2.14. A parabolic subgroup of an algebraic group is a closed subgroup containing some Borel subgroup.

Proposition 2.15. Any two Borel subgroups are conjugate.

Definition 2.16. Let $G$ be an algebraic group. The radical $R(G)$ of $G$ is the intersection of all Borel subgroups of $G$. We say that $G$ is semisimple if $R(G)=\{e\}$.

Proposition 2.17. Let $G$ be an algebraic group and $P$ a closed subgroup. Then $P$ is parabolic if and only if $G / P$ is a projective variety.

The spaces $G / P$ are called flag varieties. The flag variety $G / B$ can be identified with the set of all Borel subgroups of $G$. Since any parabolic subgroup $P \subset G$ is contained in a Borel subgroup $B$, we have a surjective $G$-equivariant (regular) map $G / B \rightarrow G / P$. Proposition 2.15 implies that $G$ acts transitively on $G / B$ and hence also on $G / P$. 
Definition 2.18. A rational representation of $G$ is a vector space $V$ together with a regular group homomorphism $G \rightarrow \mathrm{GL}(V)$.

All representations we will consider are rational.

Example 2.19. Let $\mathfrak{g}=T_{e} G$ be the tangent space at the identity of the algebraic group $G$. This is the Lie algebra of $G$. The (non-associative) algebra structure can be defined by identifying $\mathfrak{g}$ with the set of left multiplication-invariant vector fields on $G$, but we will not use this.

The Lie algebra $\mathfrak{g}$ is also a representation of $G$, called the adjoint representation. For each $g \in G$, the inner automorphism $\phi_{g}(h):=g h g^{-1}$ sends $e$ to itself, so that the differential at $e$ is an invertible linear map $d_{e} \phi_{g}: \mathfrak{g} \rightarrow \mathfrak{g}$. Thus the assignment $g \mapsto d_{e} \phi_{g}$ defines a map $G \rightarrow \mathrm{GL}(\mathfrak{g})$, which one can show is a regular group homomorphism.

Let $G$ be an semisimple affine algebraic group and choose a parabolic subgroup $P \subset G$, Borel subgroup $B \subset P$, and maximal torus $T \subset B$. Let $X(T):=\operatorname{Hom}\left(T, \mathbb{C}^{*}\right)$ (where $\mathbb{C}^{*}$ denotes the multiplicative group of nonzero complex numbers) be the weight lattice of $T$. Let $N:=\left\{g \in G \mid g t g^{-1}\right.$, for all $\left.t \in T\right\}$ be the normalizer of $T$, and let $W:=N / T$ be the Weyl group.

Proposition 2.20. Let $T \subset B \subset G$ be as above. Let $W_{P}$ the Weyl group of $P$. Then $G=B W B$ and $P=B W_{P} B$.

Over $\mathbb{C}$, any representation $V$ of $G$ decomposes into a direct sum of irreducible representations. For a maximal torus $T \subset G$, we call an element $\omega \in X(T)$ a weight of $V$ if there exists a weight vector $v \in V$ such that $t v=\omega(t) v$ for all $t \in T$. If $G$ is semisimple then $V$ is a direct sum $\bigoplus_{\omega \in X(T)} V_{\omega}$ of weight spaces for the action of $T$.

The Weyl group acts on the set of weights by setting $(w \cdot \omega)(t)=\omega\left(n t n^{-1}\right)$, where $n \in N$ is any coset representative of $w \in W$. 
The weights of the adjoint representation are called the roots of $G$. We have

Proposition 2.21. The adjoint representation has the weight space decomposition $\mathfrak{g}=\mathfrak{t} \oplus \bigoplus_{\rho \in \Phi} g_{\rho}$, where $\mathfrak{t}$ is the subalgebra of vectors of weight zero and the set of nonzero weights $\Phi \subset X(T)$ is finite.

The subalgebra $\mathfrak{t}$ in Proposition 2.21 is in fact the Lie algebra of the maximal torus $T$.

Definition 2.22. The set $\Phi \subset X(T)$ of non-zero weights of $\mathfrak{g}$ is called the root system of $G$ (given $T$ ).

Proposition 2.23. The root system $\Phi$ has the following properties:

1. $\Phi$ spans $X(T)_{\mathbb{Q}}:=X(T) \otimes \mathbb{Q}$ and does not contain 0 .

2. For each $\rho \in \Phi$ there is a reflection $s_{\rho}$ leaving $\Phi$ stable.

3. For $\rho, \sigma$ in $\Phi, s_{\rho}(\sigma)=\sigma-n_{\rho \sigma} \rho$ for some $n_{\rho \sigma} \in \mathbb{N}$.

Each reflection can be identified with an element of the Weyl group $W$, and the set $\left\{s_{\rho} \mid \rho \in \Phi\right\}$ generates $W$.

The vector space $X(T)_{\mathbb{Q}}$ admits an inner product invariant under the action of the Weyl group, which we denote $(\cdot, \cdot)$. It is called the Killing form.

Proposition 2.24. The Killing form satisfies $n_{\rho \sigma}=2(\rho, \sigma) /(\rho, \rho)$.

Fixing a Borel subgroup $B$, we have simple roots $S:=\left\{\rho_{1}, \ldots, \rho_{r}\right\}$, where $r=$ $\operatorname{dim} X(T)_{\mathbb{Q}}$.

Proposition 2.25. The set of simple roots has the following properties:

1. $S$ is a basis of $X(T)_{\mathbb{Q}}$. 
2. $W$ is generated by $\left\{s_{\rho} \mid \rho \in S\right\}$.

For $j=1, \ldots, r$, let $\omega_{j} \in X(T)$ be the unique element such that $\left(\rho_{i}, \omega_{j}\right)=\delta_{i j}$ (where $\delta_{i j}$ is the Kronecker delta function). The weights $\omega_{1}, \ldots, \omega_{r}$ are the fundamental weights.

Fix $T \subset B \subset G$, where $G$ is an affine connected semisimple complex algebraic group, $B$ a Borel subgroup, and $T$ a maximal torus. For any irreducible representation $V$, there exists a unique weight $\omega$ of $V$ such that $B V_{\omega} \subset V_{\omega}$, called the highest weight of $V$. If $V^{\prime}$ is another irreducible representation of highest weight $\omega$ then $V^{\prime} \cong V$. We denote the irreducible representation of highest weight $\omega$ by $\mathrm{L}(\omega)$. Proposition 2.26 gives an identification of the $G$-orbit of a highest weight vector in $\mathrm{L}\left(\omega_{i}\right)$ with the flag variety $G / P_{i}$.

Proposition 2.26. Let $T \subset B \subset G$ be as above. For each non-negative integer linear combination $\omega$ of the fundamental weights, there exists a unique (up to isomorphism) irreducible representation $\mathrm{L}(\omega)$ of $G$.

In particular, for each fundamental weight $\omega_{i}(1 \leq i \leq r)$ there is a unique irreducible representation of highest weight $\omega_{i}$. In this case, the stabilizer of a highest weight vector is a parabolic subgroup $P_{i} \subset B$.

We will need some facts about the representation theory of the groups $\mathrm{SL}_{n+1}(\mathbb{C})$ and $\mathrm{Sp}_{2 n}(\mathbb{C})$. We first consider the special case $\mathrm{SL}_{2}(\mathbb{C})$. For any vector space $V$ and any positive integer $m$, let $S^{m} V$ denote the $m^{\text {th }}$ symmetric power and let $\wedge^{m} V$ denote the $m^{\text {th }}$ exterior power of $V$.

Proposition 2.27. The irreducible representations of $\mathrm{SL}_{2}(\mathbb{C})$ are $S^{d} V$, where $d \in \mathbb{Z}_{+}$ and $V=\mathbb{C}^{2}$ is the defining representation. The following isomorphisms commute with 
the action of $\mathrm{SL}_{2}(\mathbb{C})$.

$$
\begin{aligned}
& S^{2}\left(S^{a} V\right) \cong \bigoplus_{k=0}^{\left\lfloor\frac{a}{2}\right\rfloor} S^{2 a-4 k} V \\
& \bigwedge^{2}\left(S^{a} V\right) \cong \bigoplus_{k=0}^{\left\lfloor\frac{a-1}{2}\right\rfloor} S^{2 a-4 k-2} V
\end{aligned}
$$

The summands on the right-hand sides of these isomorphism are irreducible.

Definition 2.28. A partition is a set of boxes (unit squares), arranged in rows and left-justified, such that as one moves from the top to the bottom the number of boxes in each row weakly decreases. We denote the partition with $k$ rows and $m_{i}$ boxes in the $i^{t h}$ row (starting from the top) by $\left(m_{1}, \ldots, m_{k}\right)$, and define $|\lambda|:=\sum_{i=1}^{k} m_{i}$.

Let $\lambda:=\left(\lambda_{1}, \ldots, \lambda_{r}\right)$ be a partition and $\lambda^{t}$ the transpose (or conjugate) partition to $\lambda$. The Schur module $[13,43] L_{\lambda} V$ is a quotient of the tensor product $\bigotimes_{i, j} V_{i, j}$, (where there is a vector space $V_{i, j} \cong V$ for each box of $\lambda$ ). It is isomorphic to the image of the homomorphism

$$
\phi_{\lambda}: \bigwedge^{\lambda_{1}} V \otimes \cdots \otimes \bigwedge^{\lambda_{r}} V \rightarrow S^{\lambda_{1}^{t}} V \otimes \cdots \otimes S^{\lambda_{r}^{t}} V
$$

defined as the composition of the exterior diagonal

$$
\delta: \bigwedge^{\lambda_{1}} V \otimes \cdots \otimes \bigwedge^{\lambda_{r}} V \rightarrow \bigotimes_{i, j} V_{i, j}
$$

followed by the multiplication map

$$
m: \bigotimes_{i, j} V_{i, j} \rightarrow S^{\lambda_{1}^{t}} V \otimes \cdots \otimes S^{\lambda_{r}^{t}} V .
$$

Proposition 2.29. Let $V=\mathbb{C}^{n+1}$ be the defining representation of $\mathrm{SL}_{n+1}(\mathbb{C})$. For each partition $\lambda=\left(n^{a_{n}}, \ldots, 1^{a_{1}}\right)$ with at most $n$ columns (that is, $\lambda$ has $a_{i}$ rows of length $i$, for each $i \in[n]$ ), the Schur module $L_{\lambda} V$ is isomorphic to the irreducible 
representation of highest weight $\omega:=\sum_{i=1}^{n} a_{i} \omega_{i}$.

The irreducible representations of $\mathrm{Sp}_{2 n}(\mathbb{C})$ have a similar description, as we will see in Chapter III, Section B.3.

\section{B. Flag varieties}

Let $G$ be a semisimple linear algebraic group. Fix a Borel subgroup $B \subset G$ and a maximal torus $T \subset B$. Let $R$ be the set of roots (determined by $T$ ), and $S:=$ $\left\{\rho_{1}, \ldots, \rho_{r}\right\}$ the simple roots (determined by $B$ ). The simple roots form an ordered basis for the Lie algebra $\mathfrak{t}$ of $T$. Let $\left\{\omega_{1}, \ldots, \omega_{r}\right\}$ be the dual basis (the fundamental weights). The Weyl group $W$ is the normalizer of $T$ modulo $T$ itself.

Let $P \subset G$ be the maximal parabolic subgroup associated to the fundamental weight $\omega \in \Omega$, let $\mathrm{L}(\omega)$ be the irreducible representation of highest weight $\omega$, and let $($,$) denote the Killing form on \mathfrak{t}$. For $\rho \in R$, let $\rho^{\vee}:=2 \rho /(\rho, \rho)$. For simplicity, assume that $\left(\omega, \rho^{\vee}\right) \leq 2$ for all $\rho \in S$ (i.e., $P$ is of classical type; see [23]). This condition implies that $\mathrm{L}(\omega)$ has $T$-fixed lines indexed by certain pairs of elements of $W / W_{P}$, called admissible (Definition 2.52).

We review these ideas in more detail for the special case of the Lagrangian Grassmannian.

\section{The Lagrangian Grassmannian}

In its natural projective embedding, the Lagrangian Grassmannian LG(n) is defined by quadratic relations which give a straightening law on a certain ordered set [9]. These relations are obtained by expressing $\mathrm{LG}(n)$ as a linear section of $\operatorname{Gr}(n, 2 n)$. While this is well-known, the author knows of no explicit derivation of these relations which do not require the representation theory of semisimple algebraic groups. We 
provide a derivation which does not rely upon representation theory (although we adopt the same notation and terminology) which will be useful when we consider the Drinfel'd Lagrangian Grassmannian, for which representation theory has yet to be successfully applied.

Set $[n]:=\{1,2, \ldots, n\}, \bar{\imath}:=-i$, and $\langle n\rangle:=\{\bar{n}, \ldots, \overline{1}, 1, \ldots n\}$. If $S$ is any set, let $\left(\begin{array}{l}S \\ k\end{array}\right)$ be the collection of subsets $\alpha=\left\{\alpha_{1}, \ldots, \alpha_{k}\right\}$ of cardinality $k$.

The Grassmannian $\operatorname{Gr}(n, 2 n)$ is the subvariety of $\mathbb{P}\left(\bigwedge^{n} \mathbb{C}^{2 n}\right)$ defined by the Plücker relations. The projective space $\mathbb{P}\left(\bigwedge^{n} \mathbb{C}^{2 n}\right)$ has Plücker coordinates indexed by the distributive lattice $\left(\begin{array}{c}{[2 n]} \\ n\end{array}\right)$. Let $\wedge$ and $\vee$ denote the meet and join in $\left(\begin{array}{c}{[2 n]} \\ n\end{array}\right)$.

Proposition 2.30 ([12, 19]). For $\alpha, \beta \in\left(\begin{array}{c}{[2 n]} \\ n\end{array}\right)$ there is a Plücker relation

$$
p_{\alpha} p_{\beta}-p_{\alpha \wedge \beta} p_{\alpha \vee \beta}+\sum_{\gamma<\alpha \wedge \beta<\alpha \vee \beta<\delta} c_{\alpha, \beta}^{\gamma, \delta} p_{\gamma} p_{\delta}=0 .
$$

The defining ideal of $\operatorname{Gr}(n, 2 n) \subset \mathbb{P}\left(\bigwedge^{n} \mathbb{C}^{2 n}\right)$ is generated by the Plücker relations.

Fix an ordered basis $\left\{e_{\bar{n}}, \ldots, e_{\overline{1}}, e_{1}, \ldots, e_{n}\right\}$ of the vector space $\mathbb{C}^{2 n}$, and let $\Omega:=\sum_{i=1}^{n} e_{\bar{\imath}} \wedge e_{i}$ be a non-degenerate alternating bilinear form. The Lagrangian Grassmannian $\operatorname{LG}(n)$ is the set of maximal isotropic subspaces (relative to $\Omega$ ) of $\mathbb{C}^{2 n}$.

Let $\left\{h_{i}:=E_{i i}-E_{\bar{\imath}} \mid i \in[n]\right\}$ be the usual basis for the Lie algebra $\mathfrak{t}$ of $T$ [13] and $\left\{h_{i}^{*} \mid i \in[n]\right\} \subset \mathfrak{t}^{*}$ the dual basis. Observe that $h_{\bar{\imath}}^{*}=-h_{i}^{*}$. The weights of any representation of $\mathrm{Sp}_{2 n}(\mathbb{C})$ are $\mathbb{Z}$-linear combinations of the fundamental weights $\omega_{i}:=h_{n-i+1}^{*}+\cdots+h_{n}^{*}$

The weights of both representations $\mathrm{L}\left(\omega_{n}\right) \subset \bigwedge^{n} \mathbb{C}^{2 n}$ and $\bigwedge^{n} \mathbb{C}^{2 n}$ are of the form $\omega=\sum_{i=1}^{n} h_{\alpha_{i}}^{*}$ for some $\alpha \in\left(\begin{array}{c}\langle n\rangle \\ n\end{array}\right)$. If $\alpha_{j}=\bar{\alpha}_{j^{\prime}}$ for some $j, j^{\prime} \in[n]$, then $h_{\alpha_{j}}^{*}=-h_{\alpha_{j^{\prime}}}^{*}$, and thus the support of $\omega$ does not contain $h_{\alpha_{j}}^{*}$. Hence the set of all such $\omega$ are indexed by elements of $\left(\begin{array}{c}\langle n\rangle \\ k\end{array}\right)$ such that $k=1, \ldots, n$ and $n-k$ is even.

Let $V$ be a vector space. For simple alternating tensors $v:=v_{1} \wedge \cdots \wedge v_{l} \in \wedge^{l} V$ 
and $\varphi:=\varphi_{1} \wedge \cdots \wedge \varphi_{k} \in \wedge^{k} V^{*}$, there is a contraction, defined by setting

$$
\varphi\lrcorner v:=\left\{\begin{array}{cl}
\sum_{I \in\left(\begin{array}{c}
{[l]} \\
k
\end{array}\right) \pm v_{1} \wedge \cdots \wedge \varphi_{1}\left(v_{i_{1}}\right) \wedge \cdots \wedge \varphi_{k}\left(v_{i_{k}}\right) \wedge \cdots \wedge v_{l},} & k \leq l \\
0, & k>l
\end{array}\right.
$$

and extending bilinearly to a map $\bigwedge^{k} V^{*} \otimes \bigwedge^{l} V \rightarrow \bigwedge^{l-k} V$. In particular, for a fixed element $\Phi \in \bigwedge^{k} V^{*}$, we obtain a linear map $\left.\Phi\right\lrcorner \bullet: \bigwedge^{l} V \rightarrow \bigwedge^{l-k} V$.

The Lagrangian Grassmannian embeds in $\operatorname{PL}\left(\omega_{n}\right)$, where $\mathrm{L}\left(\omega_{n}\right)$ is the irreducible $\mathrm{Sp}_{2 n}(\mathbb{C})$-representation of highest weight $\omega_{n}=h_{1}^{*}+\cdots+h_{n}^{*}$. By Proposition 2.31, this representation is isomorphic to the kernel of the contraction $\Omega\lrcorner \bullet: \bigwedge^{n} \mathbb{C}^{2 n} \rightarrow$ $\bigwedge^{n-2} \mathbb{C}^{2 n}$. We thus have a commutative diagram of injective maps:

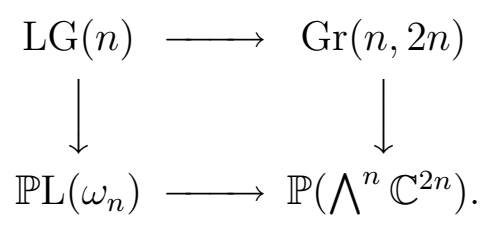

The next proposition implies that $\mathrm{LG}(n)=\operatorname{Gr}(n, 2 n) \cap \mathbb{P L}\left(\omega_{n}\right)$.

Proposition 2.31. The dual of the contraction map

$$
\Omega\lrcorner \bullet: \bigwedge^{n} \mathbb{C}^{2 n} \rightarrow \bigwedge^{n-2} \mathbb{C}^{2 n}
$$

is the multiplication map

$$
\Omega \wedge \bullet: \bigwedge^{n-2} \mathbb{C}^{2 n^{*}} \rightarrow \bigwedge^{n} \mathbb{C}^{2 n^{*}}
$$

Furthermore, the irreducible representation $\mathrm{L}\left(\omega_{n}\right)$ is defined by the vanishing of the linear forms

$$
L_{n}:=\left\langle\Omega \wedge e_{\alpha_{1}}^{*} \wedge \cdots \wedge e_{\alpha_{n-2}}^{*} \mid \alpha \in\left(\begin{array}{c}
\langle n\rangle \\
n-2
\end{array}\right)\right\rangle
$$

These linear forms cut out LG(n) scheme-theoretically in $\operatorname{Gr}(n, 2 n)$. Dually, $\mathrm{L}\left(\omega_{n}\right)=$ $\operatorname{ker}(\Omega\lrcorner \bullet)$ 
Proof. Statement (1) is straightforward, and (2) can be found in [43, Ch. 3, Exercise 1; Ch. 6, Exercise 24].

Since the linear forms in $L_{n}$ are supported on variables indexed by $\alpha \in\left(\begin{array}{c}\langle n\rangle \\ n\end{array}\right)$ such that $\{\bar{\imath}, i\} \in \alpha$ for some $i \in[n]$, the set of complementary variables is linearly independent. These are indexed by the set $\mathcal{P}_{n}$ of admissible elements of $\left(\begin{array}{c}\langle n\rangle \\ n\end{array}\right)$

$$
\mathcal{P}_{n}:=\left\{\alpha \in\left(\begin{array}{c}
\langle n\rangle \\
n
\end{array}\right) \mid i \in \alpha \Leftrightarrow \bar{\imath} \notin \alpha\right\}
$$

and have a nice description in terms of partitions (Definition 2.28).

Consider the lattice $\mathbb{Z}^{2}$ with coordinates $(a, b)$ corresponding to the point $a$ units below the origin and $b$ units to the right of the origin. Given an increasing sequence $\alpha \in\left(\begin{array}{c}\langle n\rangle \\ n\end{array}\right)$, let $[\alpha]$ be the lattice path beginning at $(n, 0)$, ending at $(0, n)$, and whose $i^{\text {th }}$ step is vertical if $i \in \alpha$ and horizontal if $i \notin \alpha$. We can associate a partition to $\alpha$ by taking the boxes lying in the region bounded by the coordinate axes and $[\alpha]$. For instance, the sequence $\alpha=\overline{4} \overline{2} 23 \in\left(\begin{array}{c}\langle 4\rangle \\ 4\end{array}\right)$ is associated to the partition shown in Figure 1.

Proposition 2.32. The bijection between increasing sequences and partitions induces a bijection between sequences $\alpha$ which do not contain both $i$ and $\bar{\imath}$ for any $i \in[n]$, and partitions which lie inside the $n \times n$ square $\left(n^{n}\right)$ and are symmetric with respect to reflection about the diagonal $\{(a, a) \mid a \in \mathbb{Z}\} \subset \mathbb{Z}^{2}$.

Proof. $\mathcal{P}_{n}$ consists of the $\alpha \in\left(\begin{array}{c}\langle n\rangle \\ n\end{array}\right)$ which are fixed upon negating each element of $\alpha$ and taking the complement in $\langle n\rangle$. On the other hand, the composition of these two operations corresponds to reflecting the associated diagram about the diagonal. 


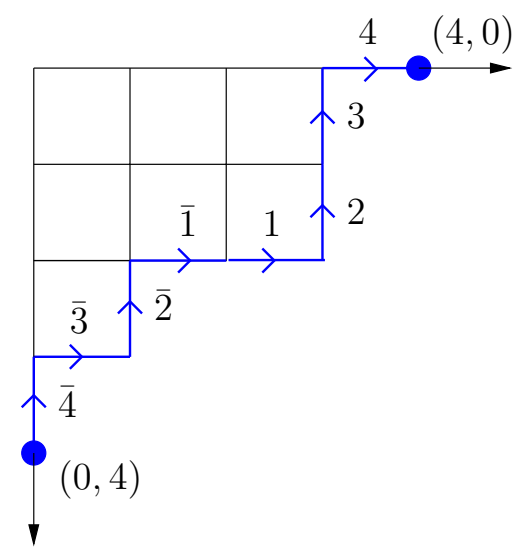

Fig. 1. The partition $(3,3,1)$ associated to $\overline{4} \overline{2} 23$.

Remark 2.33. We will use an element of $\left(\begin{array}{c}\langle n\rangle \\ n\end{array}\right)$ and its associated partition interchangeably. We denote by $\alpha^{t}$ the transpose partition obtained by reflecting $\alpha$ about the diagonal in $\mathbb{Z}^{2}$. As a sequence, $\alpha^{t}$ is the complement of $\left\{\bar{\alpha}_{1}, \ldots, \bar{\alpha}_{n}\right\} \subset\langle n\rangle$.

Definition 2.34. The Lagrangian involution is the map $\tau: p_{\alpha} \mapsto \sigma_{\alpha} p_{\alpha^{t}}$, where $\alpha^{t}$ is the sequence obtained by negating each element of the complement of $\alpha$, and $\sigma_{\alpha}:=\operatorname{sgn}\left(\alpha_{+}^{c}, \alpha_{+}\right) \operatorname{sgn}\left(\alpha_{-}, \alpha_{-}^{c}\right) \in\{ \pm 1\}$.

We denote by $\alpha_{+}$(respectively, $\alpha_{-}$) the subset of positive (respectively, negative) elements of $\alpha$. For example, if $\alpha=\overline{4} \overline{1} 23$, then $\alpha_{+}=23$ and $\alpha_{-}=\overline{4} \overline{1}$.

The Grassmannian $\operatorname{Gr}(n, 2 n)$ has a natural geometric involution $\bullet^{\perp}: \operatorname{Gr}(n, 2 n) \rightarrow$ $\operatorname{Gr}(n, 2 n)$ sending an $n$-plane $U$ to its orthogonal complement $U^{\perp}:=\left\{u \in \mathbb{C}^{2 n} \mid\right.$ $\Omega\left(u, u^{\prime}\right)=0$, for all $\left.u^{\prime} \in U\right\}$ with respect to $\Omega$. The next proposition relates $\bullet^{\perp}$ to the Lagrangian involution. 
Proposition 2.35. The map $\bullet^{\perp}: \operatorname{Gr}(n, 2 n) \rightarrow \operatorname{Gr}(n, 2 n)$ expressed in Plücker coordinates coincides with the Lagrangian involution:

$$
\left[p_{\alpha} \mid \alpha \in\left(\begin{array}{c}
\langle n\rangle \\
n
\end{array}\right)\right] \mapsto\left[\sigma_{\alpha} p_{\alpha^{t}} \mid \alpha \in\left(\begin{array}{c}
\langle n\rangle \\
n
\end{array}\right)\right]
$$

In particular, the relation $p_{\alpha}-\sigma_{\alpha} p_{\alpha^{t}}$ holds on $\mathrm{LG}(n)$.

Proof. The set of $n$-planes in $\mathbb{C}^{2 n}$ which do not meet the span of the first $n$ standard basis vectors is open and dense in $\operatorname{Gr}(n, 2 n)$. Any such $n$-plane is the row space of an $n \times 2 n$ matrix

$$
Y:=(I \mid X)
$$

where $I$ is the $n \times n$ identity matrix and $X$ is some $n \times n$ matrix. We work in the affine coordinates given by the entries in $X$. For $\alpha \in\left(\begin{array}{c}\langle n\rangle \\ n\end{array}\right)$, we denote the $\alpha^{t h}$ minor of $Y$ by $p_{\alpha}(Y)$. For a set of indices $\alpha=\left\{\alpha_{1}, \ldots, \alpha_{k}\right\} \subset[n]$, let $\alpha^{c}:=[n] \backslash \alpha$ be the complement, $\alpha^{\prime}:=\left\{n-\alpha_{k}+1, \ldots, n-\alpha_{1}+1\right\}$, and $\bar{\alpha}:=\left\{\bar{\alpha}_{1}, \ldots, \bar{\alpha}_{k}\right\}$. Via the correspondence between partitions and sequences (Proposition 2.32), $\alpha^{t}=\bar{\alpha}^{c}$.

We claim that $\bullet^{\perp}$ reflects $X$ along the antidiagonal. To see this, we simply observe how the rows of $Y$ pair under $\Omega$. For $u, v \in \mathbb{C}^{n}$, we denote the concatenation by $(u, v) \in \mathbb{C}^{2 n}$. Let $r_{i}:=\left(e_{i}, v_{i}\right) \in \mathbb{C}^{2 n}$ be the $i^{\text {th }}$ row of $Y$. For $k \in\langle n\rangle$, we let $r_{i k} \in \mathbb{C}$ be the $k^{t h}$ component of $r_{i}$. Then, for $i, j \in\langle n\rangle$,

$$
\begin{aligned}
\Omega\left(r_{i}, r_{j}\right) & =\left(e_{i}, v_{i}\right) \cdot\left(-v_{j}, e_{j}\right)^{t} \\
& =r_{i, n-j+1}-r_{j, i}
\end{aligned}
$$

It follows that the effect of $\tau$ on the minor $X_{\rho, \gamma}$ of $X$ given by row indices $\rho$ and column indices $\gamma$ is

$$
\tau(X)_{\rho, \gamma}=X_{\gamma^{\prime}, \rho^{\prime}}
$$


Let $\alpha=\bar{\epsilon} \cup \phi \in\left(\begin{array}{c}\langle n\rangle \\ n\end{array}\right)$, where $\epsilon$ and $\phi$ are subsets of $[n]$. Combining this description of $\tau$ with the identity

$$
p_{\alpha}(Y)=\operatorname{sgn}\left(\epsilon^{c}, \epsilon\right) X_{\left(\epsilon^{c}\right)^{\prime}, \phi}
$$

we have

$$
\begin{aligned}
p_{\alpha}(\tau(Y)) & =\operatorname{sgn}\left(\epsilon^{c}, \epsilon\right) \tau(X)_{\left(\epsilon^{c}\right)^{\prime}, \phi} \\
& =\operatorname{sgn}\left(\epsilon^{c}, \epsilon\right) X_{\phi^{\prime}, \epsilon^{c}} \\
& =\operatorname{sgn}\left(\epsilon^{c}, \epsilon\right) \operatorname{sgn}\left(\phi, \phi^{c}\right) p_{\left(\bar{\phi}^{c}, \epsilon^{c}\right)}(Y) \\
& =\sigma_{\alpha} p_{\alpha^{t}}(Y) .
\end{aligned}
$$

It follows that the relation $p_{\alpha}-\sigma_{\alpha} p_{\alpha^{t}}=0$ holds on a dense open subset and hence identically on all of $\mathrm{LG}(n)$.

It follows from Proposition 2.35 that the system of linear equations

$$
L_{n}^{\prime}:=\left\langle p_{\alpha}-\sigma_{\alpha} p_{\alpha^{t}} \mid \alpha \in\left(\begin{array}{c}
\langle n\rangle \\
n
\end{array}\right)\right\rangle
$$

defines $\operatorname{LG}(n) \subset \operatorname{Gr}(n, 2 n)$ set-theoretically. Since $\operatorname{LG}(n)$ lies in no hyperplane of $\operatorname{PL}\left(\omega_{n}\right), L_{n}^{\prime}$ is a linear subspace of the span $L_{n}$ of the defining equations of $\mathrm{L}\left(\omega_{n}\right) \subset \bigwedge^{n} \mathbb{C}^{2 n}$. The generators of $L_{n}^{\prime}$ above suggests that homogeneous coordinates for the Lagrangian Grassmannian should be indexed by some sort of quotient (which we will call $\mathcal{D}_{n}$ ) of the poset $\mathcal{P}_{n}$. The correct notion of quotient is that of a doset (Definition 2.43). An important set of representatives for $\mathcal{D}_{n}$ in $\mathcal{P}_{n}$ is given in Proposition 2.38 .

By Proposition 2.32, we may identify elements of $\left(\begin{array}{c}\langle n\rangle \\ n\end{array}\right)$ with partitions lying in the $n \times n$ square $\left(n^{n}\right)$, and $\mathcal{P}_{n}$ with the set of symmetric partitions. Define a map $\pi_{n}:\left(\begin{array}{c}\langle n\rangle \\ n\end{array}\right) \rightarrow \mathcal{P}_{n} \times \mathcal{P}_{n}$ by $\pi_{n}(\alpha):=\left(\alpha \wedge \alpha^{t}, \alpha \vee \alpha^{t}\right)$. Let $\mathcal{D}_{n}$ be the image of $\pi_{n}$. It is called the set of admissible pairs. It is a subset of $\mathcal{O}_{\mathcal{P}_{n}}:=\left\{(\alpha, \beta) \in \mathcal{P}_{n} \times \mathcal{P}_{n} \mid \alpha \leq \beta\right\}$. 
The image of $\mathcal{P}_{n} \subset\left(\begin{array}{c}\langle n\rangle \\ n\end{array}\right)$ under $\pi_{n}$ is the diagonal $\Delta_{\mathcal{P}_{n}} \subset \mathcal{P}_{n} \times \mathcal{P}_{n}$.

To show that $\mathcal{D}_{n}$ indexes coordinates on $\mathrm{LG}(n)$, we will work with a convenient set of representatives of the fibers of $\pi_{n}$. The fiber of $(\alpha, \beta) \in \mathcal{D}_{n}$ can be described as follows. The lattice paths $[\alpha]$ and $[\beta]$ must meet at the diagonal. Since $\alpha$ and $\beta$ are symmetric, they are determined by the segments of their associated paths to the right and above the diagonal. Let $\Pi(\alpha, \beta)$ be the set of boxes bounded by these segments. Taking $n=4$ for example, $\Pi(\overline{4} \overline{2} 13, \overline{3} \overline{1} 24)$ consists of the two shaded boxes above the diagonal in Figure 2. The lattice path [ $\overline{4} \overline{2} 13]$ is above and to the left of the path $[\overline{3} \overline{1} 24]$.

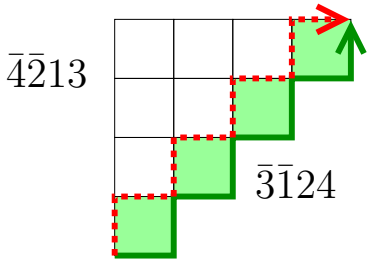

Fig. 2. The paths associated to $\overline{4} \overline{2} 13$ and $\overline{3} \overline{1} 24$ in $\mathcal{P}_{4}$.

A subset $S \subset\left(n^{n}\right)$ of boxes is disconnected if $S=S_{1} \sqcup S_{2}$ and no box of $S_{1}$ shares an edge with a box of $S_{2}$. A subset $S$ is connected if it is not disconnected. Let $\Pi(\alpha, \beta)=\bigsqcup_{i=1}^{k} S_{i}$ be the decomposition of $\Pi(\alpha, \beta)$ into its connected components.

Any element $\gamma$ of the fiber $\pi_{n}^{-1}(\alpha, \beta)$ is obtained by choosing a subset $I \subset[k]$ 
and setting

$$
\gamma=\alpha \cup\left(\bigcup_{i \in I} S_{i}^{t}\right) \cup\left(\bigcup_{i \notin I} S_{i}\right)
$$

The elements of $\pi_{n}^{-1}(\overline{4} \overline{2} 13, \overline{3} \overline{1} 24)$ are shown in Figure 3.

For any diagram $\alpha \subseteq\left(n^{n}\right)$, let $\alpha_{+} \subseteq \alpha$ be the subset of $\alpha$ on or above the main diagonal, and $\alpha_{-} \subseteq \alpha$ the subset of $\alpha$ on or below the main diagonal. Equivalently, $\alpha$ is a strictly increasing subsequence of $(\bar{n}, \ldots, \overline{1}, 1, \ldots, n)$ of length $n, \alpha_{+}$is the subset of positive elements of $\alpha$, and $\alpha_{-}$is the set of negative elements. Similarly, let $\Pi_{+}(\alpha, \beta) \subset \Pi(\alpha, \beta)$ be the set of boxes above the diagonal.

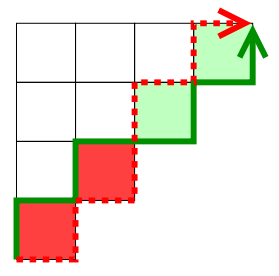

$\overline{4} \overline{2} 24$ and $\overline{3} \overline{1} 13$

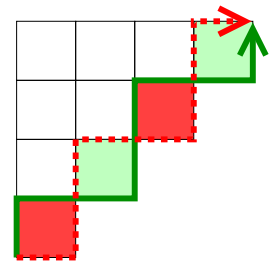

$\overline{4} \overline{1} 14$ and $\overline{3} \overline{2} 23$

Fig. 3. Elements of $\pi_{n}^{-1}(\overline{4} \overline{2} 13, \overline{3} \overline{1} 24)$.

The following definition is essential.

Definition 2.37. A partition $\alpha$ Northeast if $\alpha_{-}^{t} \subseteq \alpha_{+}$and Southwest if its transpose is Northeast. 
For example, $\overline{4} \overline{2} 24 \in \mathcal{P}_{4}$ is Northeast while $\overline{4} \overline{1} 14 \in \mathcal{P}_{4}$ is neither Northeast nor Southwest.

We summarize these ideas in the following proposition.

Proposition 2.38. Let $(\alpha, \beta) \in \mathcal{D}_{n}$ be an element of the image of $\pi_{n}$. Then $\pi_{n}^{-1}(\alpha, \beta)$ is in bijection with the set $\mathbf{2}^{\Pi_{+}(\alpha, \beta)}$ of subsets of connected components of $\Pi_{+}(\alpha, \beta)$. There exists a unique Northeast element of $\pi_{n}^{-1}(\alpha, \beta)$; namely, the element corresponding to all the connected components. Similarly, there is a unique Southwest element corresponding to the empty set of components.

Example 2.39. The Lagrangian Grassmannian $\operatorname{LG}(4) \subset \operatorname{Gr}(4,8)$ is defined by the linear ideal $L_{4}$. From the explicit generators given in Proposition 2.31, it is evident that $L_{4} \subset \bigwedge^{4} \mathbb{C}^{8 *}$ is spanned by weight vectors. For example, the generators of $L_{4} \cap\left(\bigwedge^{4} \mathbb{C}^{8^{*}}\right)_{0}$ are vectors of weight zero:

$$
\begin{aligned}
& \Omega \wedge p_{\overline{1} 1}=p_{\overline{4} \overline{1} 14}+p_{\overline{3} \overline{1} 13}+p_{\overline{2} \overline{1} 12} \\
& \Omega \wedge p_{\overline{2} 2}=p_{\overline{4} \overline{2} 24}+p_{\overline{3} \overline{2} 23}+p_{\overline{2} \overline{1} 12} \\
& \Omega \wedge p_{\overline{3} 3}=p_{\overline{4} \overline{3} 34}+p_{\overline{3} \overline{2} 23}+p_{\overline{3} \overline{1} 13} \\
& \Omega \wedge p_{\overline{4} 4}=p_{\overline{4} \overline{3} 34}+p_{\overline{4} \overline{2} 24}+p_{\overline{4} \overline{1} 14}
\end{aligned}
$$

The following linear forms lie in the span of the right-hand side of (2.40):

$$
\begin{gathered}
p_{\overline{2} \overline{1} 12}+p_{\overline{4} \overline{3} 34} \\
p_{\overline{3} \overline{1} 13}+p_{\overline{4} \overline{2} 24} \quad p_{\overline{3} \overline{2} 23}+p_{\overline{4} \overline{1} 14} \\
p_{\overline{4} \overline{1} 14}+p_{\overline{4} \overline{2} 24}+p_{\overline{4} \overline{3} 34}
\end{gathered}
$$

Three of the linear forms in (2.41) are supported on a pair $\left\{p_{\alpha}, p_{\alpha^{t}}\right\}$, and the remaining linear form expresses the Plücker coordinate $p_{\overline{4} \overline{1} 14}$ as a linear combination of coordinates indexed by Northeast partitions (in general, this follows from Lemma 3.10). Since each pair $\left\{p_{\alpha}, p_{\alpha^{t}}\right\}$ is incomparable, there is a Plücker relation which, after 
reduction by the linear forms $(2.40)$, takes the form

$$
\pm p_{\alpha}^{2}-p_{\beta} p_{\gamma}+\text { lower order terms }
$$

where $\beta:=\alpha \wedge \alpha^{t}$ and $\gamma:=\alpha \vee \alpha^{t}$ are respectively the meet and join of $\alpha$ and $\alpha^{t}$. Defining $p_{(\beta, \gamma)}:=p_{\alpha}=\sigma_{\alpha} p_{\alpha^{t}}$ we can regard such an equation as giving a rule for rewriting $p_{(\beta, \gamma)}^{2}$ as a linear combination of monomials supported on a chain. These facts are proven in general in Chapter III.

\section{Spaces of algebraic maps}

The flag variety $G / P$ embeds in $\mathbb{P L}(\omega)$ as the orbit of a highest weight vector. Define the degree of a rational map $f: \mathbb{P}^{1} \rightarrow G / P$ to be its degree as a rational map into $\mathbb{P L}(\omega)$.

Let $\mathcal{M}_{d}(G / P)$ be the space of algebraic maps of degree $d$ from $\mathbb{P}^{1}$ into $G / P$. If $P$ is of classical type then the set $\mathcal{D}$ of admissible pairs indexes the homogeneous coordinates on $\mathbb{P L}(\omega)$ (see Definition 2.52, and for more background [35, 23]). Therefore, any map $f \in \mathcal{M}_{d}(G / P)$ can be expressed as $f:[s, t] \mapsto\left[p_{w}(s, t) \mid w \in \mathcal{D}\right]$, where $\mathcal{D}$ is the set of admissible pairs and $p_{w}(s, t)$ is a homogeneous form of degree d. This leads to an embedding of $\mathcal{M}_{d}(G / P)$ in $\mathbb{P}\left(S^{d} \mathbb{C}^{2} \otimes \mathrm{L}(\omega)\right)$, where $S^{d} \mathbb{C}^{2}$ is the space of homogeneous polynomials of degree $d$ in two variables. Coordinate functions on $S^{d} \mathbb{C}^{2} \otimes \mathrm{L}(\omega)$ are indexed by $\left\{w^{(a)} \mid w \in \mathcal{D}, a=0, \ldots, d\right\}$, a disjoint union of $d+1$ copies of $\mathcal{D}$. The closure of $\mathcal{M}_{d}(G / P) \subset \mathbb{P}\left(S^{d} \mathbb{C}^{2} \otimes \mathrm{L}(\omega)\right)$ is called the Drinfel'd compactification and denoted $\mathcal{Q}_{d}(G / P)$. This definition is due to V. Drinfel'd, dating from the mid-1980s. Drinfel'd never published this definition himself; to the author's knowledge its first appearance in print was in [36]; see also [22].

Let $G=\mathrm{SL}_{n}(\mathbb{C})$ and $P$ be the maximal parabolic subgroup stabilizing a $k$ - 
dimensional subspace, so that $G / P=\operatorname{Gr}(k, n)$. In this case we denote the Drinfel'd Grassmannian $\mathcal{Q}_{d}(G / P)$ by $\mathcal{Q}_{d}(k, n)$. In [41] it is shown that the homogeneous coordinate ring of $\mathcal{Q}_{d}(k, n)$ is an algebra with straightening law on a distributive lattice $\left(\begin{array}{c}{[n]} \\ k\end{array}\right)_{d}$, hence normal, Cohen-Macaulay, and Koszul, and its ideal $I_{k, n-k}^{d}$ has a quadratic Gröbner basis consisting of the straightening relations. The ideal $I_{k, n-k}^{d}$ is a subset of the polynomial ring $\mathbb{C}\left[\left(\begin{array}{c}{[n]} \\ k\end{array}\right)_{d}\right]$ whose variables are indexed by elements of $\left(\begin{array}{c}{[n]} \\ k\end{array}\right)_{d}:=\left\{\alpha^{(a)} \mid \alpha \in\left(\begin{array}{c}{[n]} \\ k\end{array}\right), 0 \leq a \leq d\right\}$. The partial order on $\left(\begin{array}{c}{[n]} \\ k\end{array}\right)_{d}$ is defined by $\alpha^{(a)} \leq \beta^{(b)}$ if and only if $a \leq b$ and $\alpha_{i} \leq \beta_{b-a+i}$ for $i=1, \ldots, k-b+a$.

Note that taking $d=0$ above, one recovers the classical Bruhat order on $\left(\begin{array}{c}{[n]} \\ k\end{array}\right)$. For an arbitrary semisimple algebraic group, this is an ordering on the set of maximal coset representatives of the quotient of the Weyl group by the Weyl group of a parabolic subgroup.

Suppose that $d=\ell k+q$ for positive integers $\ell$ and $q$, and let $X=\left(x_{i j}\right)_{1 \leq i, j \leq n}$ be a matrix of polynomials with $x_{i j}=x_{i j}^{\left(k_{i}\right)} t^{k_{i}}+\cdots+x_{i j}^{(1)} t+x_{i j}^{(0)}, k_{i}=\ell+1$ for $i \leq q$, and $k_{i}=\ell$ for $i>q$. The ideal $I_{k, n-k}^{d}$ is the kernel of the map $\varphi: \mathbb{C}\left[\left(\begin{array}{c}{[n]} \\ k\end{array}\right)_{d}\right] \rightarrow \mathbb{C}[X]$ sending the variable $p_{\alpha}^{(a)}$ indexed by $\alpha^{(a)}$ to the coefficient of $t^{a}$ in the maximal minor of $X$ whose columns are indexed by $\alpha$.

The main results of [41] follow from the next theorem. Given any distributive lattice, we denote by $\wedge$ and $\vee$, respectively, the meet and join. The symbol $\wedge$ will also be used for exterior products of vectors, but the meaning should always be clear from the context.

Theorem 2.42 ([41]). Let $\gamma, \epsilon$ be a pair of incomparable variables in the poset $\left(\begin{array}{c}{[n]} \\ k\end{array}\right)_{d}$. There is a quadratic polynomial $S(\gamma, \epsilon)$ in the kernel of $\varphi: \mathbb{C}\left[\left(\begin{array}{c}{[n]} \\ k\end{array}\right)_{d}\right] \rightarrow \mathbb{C}[X]$ whose first two monomials are

$$
p_{\gamma} p_{\epsilon}-p_{\gamma \wedge \epsilon} p_{\gamma \vee \epsilon} .
$$


Moreover, if $\lambda p_{\beta} p_{\alpha}$ is any non-initial monomial in $S(\gamma, \epsilon)$, then $\gamma, \epsilon$ lies in the interval $[\beta, \alpha]=\left\{\gamma \in\left(\begin{array}{c}{[n]} \\ k\end{array}\right)_{d} \mid \beta \leq \gamma \leq \alpha\right\}$.

The quadratic polynomials $S(\gamma, \epsilon)$ in fact form a Gröbner basis for the ideal they generate. It is shown in [41] that there exists a toric (SAGBI) deformation taking $S(\gamma, \epsilon)$ to its initial form $p_{\gamma} p_{\epsilon}-p_{\gamma \wedge \epsilon} p_{\gamma \vee \epsilon}$.

Our main goal is to extend this result to the Lagrangian Drinfel'd Grassmannian $L \mathcal{Q}_{d}(n):=\mathcal{Q}_{d}(\operatorname{LG}(n))$ parametrizing rational curves of degree $d$ in the Lagrangian Grassmannian.

The role of flag varieties in classical enumerative geometry was described in Chapter I. Drinfel'd flag varieties are similarly useful in quantum cohomology, which deals with the enumerative geometry of curves in a flag variety. We illustrate this for the case of the Drinfel'd Grassmannian [39, 41].

Let $X_{\alpha} \subset \operatorname{Gr}(k, n)$ be a Schubert variety and $s \in \mathbb{P}^{1}$ a point. An element of the Drinfel'd Grassmannian $E(t) \in \mathcal{Q}_{d}(k, n)$, is a parametrized curve $t \mapsto E(t) \in \operatorname{Gr}(k, n)$ of degree $d$. Given a non-negative integer $a \leq d$, the set of all $E(t) \in \mathcal{Q}_{d}(k, n)$ such that $E(t)$ meets $X_{\alpha}$ to order $a$ at the point $s$ is a Schubert variety $X_{\alpha^{(a)}}(s)$. When $s=0 \in \mathbb{P}^{1}$, this coincides with the definition given in Section $\mathrm{D}$, below. The realization of the coordinate ring of a Drinfel'd flag variety (and its Schubert varieties) allows one to compute the degree of a Schubert variety, thus recovering certain intersection numbers in quantum cohomology.

D. Algebras with straightening laws

The following definitions are fundamental.

Let $\mathcal{P}$ be a poset, $\Delta_{\mathcal{P}}$ the diagonal in $\mathcal{P} \times \mathcal{P}$, and $\mathcal{O}_{\mathcal{P}}:=\{(\alpha, \beta) \in \mathcal{P} \times \mathcal{P} \mid \alpha \leq \beta\}$ the subset of $\mathcal{P} \times \mathcal{P}$ defining the order relation on $\mathcal{P}$. 
Definition 2.43. A doset on $\mathcal{P}$ is a set $\mathcal{D}$ such that $\Delta_{\mathcal{P}} \subset \mathcal{D} \subset \mathcal{O}_{\mathcal{P}}$, and if $\alpha \leq \beta \leq \gamma$, then $(\alpha, \gamma) \in \mathcal{D}$ if and only if $(\alpha, \beta) \in \mathcal{D}$ and $(\beta, \gamma) \in \mathcal{D}$. The ordering on $\mathcal{D}$ is given by $(\alpha, \beta) \leq(\gamma, \delta)$ if and only if $\beta \leq \gamma$ in $\mathcal{P}$. We refer to $\mathcal{P}$ as the underlying poset.

Remark 2.44. A doset is not usually a poset: the ordering is non-reflexive except in the trivial case when $\mathcal{D}=\Delta_{\mathcal{P}}$. In any case, note that we may regard $\mathcal{P} \cong \Delta_{\mathcal{P}}$ as a subset of $\mathcal{D}$.

The Hasse diagram of a doset $\mathcal{D}$ on $\mathcal{P}$ is obtained from the Hasse diagram of $\mathcal{P} \subset \mathcal{D}$ by drawing a double line for each cover $\alpha \lessdot \beta$ such that $(\alpha, \beta)$ is in $\mathcal{D}$. The defining property of a doset implies that we can recover all the information in the doset from its Hasse diagram. See Figure 6 for an example.

Loosely, an algebra with straightening law is an algebra generated by elements $\left\{p_{\alpha} \mid \alpha \in \mathcal{D}\right\}$ indexed by a (finite) doset with a basis consisting of standard monomials supported on a chain. That is, a monomial is standard if it has the form $p_{\left(\alpha_{1}, \beta_{1}\right)} \cdots p_{\left(\alpha_{k}, \beta_{k}\right)}$, where $\alpha_{1} \leq \beta_{1} \leq \alpha_{2} \leq \beta_{2} \leq \cdots \leq \alpha_{k} \leq \beta_{k}$. Furthermore, monomials which are not standard are subject to so-called straightening relations, as described in the following definition.

Definition 2.45. Let $\mathcal{D}$ be a doset. A graded $\mathbb{C}$-algebra $A=\bigoplus_{q \geq 0} A_{q}$ is an algebra with straightening law on $\mathcal{D}$ if there is an injection $\mathcal{D} \ni(\alpha, \beta) \mapsto p_{(\alpha, \beta)} \in A$ such that:

1. $\left\{p_{(\alpha, \beta)} \mid(\alpha, \beta) \in \mathcal{D}\right\}$ generates $A$.

2. The set of standard monomials are a $\mathbb{C}$-basis of $A$.

3. For any monomial $m=p_{\left(\alpha_{1}, \beta_{1}\right)} \cdots p_{\left(\alpha_{k}, \beta_{k}\right)},\left(\alpha_{i}, \beta_{i}\right) \in \mathcal{D}$ for $i=1, \ldots, k$, if

$$
m=\sum_{j=1}^{N} c_{j} p_{\left(\alpha_{j 1}, \beta_{j 1}\right)} \cdots p_{\left(\alpha_{j k}, \beta_{j k}\right)}
$$


is the unique expression of $m$ as a linear combination of distinct standard monomials, then the sequence $\left(\alpha_{j 1} \leq \beta_{j 1} \leq \cdots \leq \alpha_{j k} \leq \beta_{j k}\right)$ is lexicographically smaller than $\left(\alpha_{1} \leq \beta_{1} \leq \cdots \leq \alpha_{k} \leq \beta_{k}\right)$. That is, if $\ell \in[2 k]$ is minimal such that $\alpha_{j \ell} \neq \alpha_{\ell}$, then $\alpha_{j \ell}<\alpha_{\ell}$.

4. If $\alpha_{1} \leq \alpha_{2} \leq \alpha_{3} \leq \alpha_{4}$ are such that for some permutation $\sigma \in S_{4}$ we have $\left(\alpha_{\sigma(1)}, \alpha_{\sigma(2)}\right) \in \mathcal{D}$ and $\left(\alpha_{\sigma(3)}, \alpha_{\sigma(4)}\right) \in \mathcal{D}$, then

$$
p_{\left(\alpha_{\sigma(1)}, \alpha_{\sigma(2)}\right)} p_{\left(\alpha_{\sigma(3)}, \alpha_{\sigma(4)}\right)}= \pm p_{\left(\alpha_{1}, \alpha_{2}\right)\left(\alpha_{3}, \alpha_{4}\right)}+\sum_{i=1}^{N} r_{i} m_{i}
$$

where the $m_{i} \in A_{2}$ are standard monomials distinct from $p_{\left(\alpha_{1}, \alpha_{2}\right)} p_{\left(\alpha_{3}, \alpha_{4}\right)}$.

The ideal of straightening relations is generated by homogeneous quadratic forms in the $p_{\alpha}(\alpha \in \mathcal{D})$, so we may consider the projective variety $X:=\operatorname{Proj} A$ they define. For each $\alpha \in \mathcal{P}$, we have the Schubert variety

$$
X_{\alpha}:=\left\{x \in X \mid p_{(\beta, \gamma)}(x)=0 \text { for } \gamma \not \leq \alpha\right\}
$$

and the dual Schubert variety

$$
X^{\alpha}:=\left\{x \in X \mid p_{(\beta, \gamma)}(x)=0 \text { for } \beta \nsupseteq \alpha\right\} .
$$

Let us recall the geometry of these varieties for the Grassmannian of $k$-planes in $\mathbb{C}^{n}$, whose coordinate ring is an algebra with straightening law on the poset $\left(\begin{array}{c}{[n]} \\ k\end{array}\right)$.

For each $i \in[n]$, set $F_{i}:=\left\langle e_{1}, \ldots, e_{i}\right\rangle$ and $F_{i}^{\prime}:=\left\langle e_{n}, \ldots, e_{n-i+1}\right\rangle$, where $\langle\cdots\rangle$ denotes linear span and $\left\{e_{1}, \ldots, e_{n}\right\}$ is the standard basis of $\mathbb{C}^{n}$. We call $F_{\bullet}:=\left\{F_{1} \subset\right.$ $\left.\cdots \subset F_{n}\right\}$ the standard coordinate flag, and $F_{\bullet}^{\prime}:=\left\{F_{1}^{\prime} \subset \cdots \subset F_{n}^{\prime}\right\}$ the opposite flag.

For $\alpha=\left\{1 \leq \alpha_{1}<\cdots<\alpha_{k} \leq n\right\} \in\left(\begin{array}{c}{[n]} \\ k\end{array}\right)$, define $X_{\alpha}:=\{E \in \operatorname{Gr}(k, n) \mid \operatorname{dim}(E \cap$ $\left.\left.F_{n-\alpha_{i}+1}^{\prime}\right) \leq i, i=1, \ldots, k\right\}$ and $X^{\alpha}:=\left\{E \in \operatorname{Gr}(k, n) \mid \operatorname{dim}\left(E \cap F_{\alpha_{i}}^{\prime}\right) \leq i, i=1, \ldots, k\right\}$.

We represent any $k$-plane $E \in \operatorname{Gr}(k, n)$ as the row space of a $k \times n$ matrix. 
Furthermore, any such $k$-plane $E$ is the row space of a unique reduced row echelon matrix. The Schubert variety $X_{\alpha}$ consists of precisely the $k$-planes $E$ such that the pivot in row $i$ is weakly to the right of column $n-\alpha_{i}+1$. Since the Plücker coordinate $p_{\alpha}(E)$ is just the maximal minor of this matrix, we see that $E \in X_{\alpha}$ if and only if $p_{\beta}(E)=0$ for all $\beta \not \leq \alpha$; hence our definition of $X_{\alpha}$ for the Grassmannian agrees with the general definition above.

Schubert varieties are useful and natural tools for studying algebras with straightening law, as we will see in Chapter III.

1. Hilbert series of an algebra with straightening law

We compute the Hilbert series of an algebra with straightening law $A$ on a doset, and thus obtain formulas for the dimension and degree of Proj $A$. Let $\mathcal{P}$ be a poset and $\mathcal{D}$ a doset on $\mathcal{P}$. Assume that all maximal chains in $\mathcal{D}$ (respectively, $\mathcal{P}$ ) have the same length $d$ (respectively, $p$ ).

We first compute the Hilbert series of $A$ with respect to a suitably chosen fine grading of $A$. Namely, $A$ is graded by the elements of a semigroup, defined as follows.

Monomials in $\mathbb{C}[\mathcal{D}]$ are determined by their exponent vectors. We can therefore identify the set of such monomials with the semigroup $\mathbb{N}^{\mathcal{D}}$. Define the weight map $\mathbf{w}: \mathbb{N}^{\mathcal{D}} \rightarrow \mathbb{Q}^{\mathcal{P}}$ by setting $\mathbf{w}(\alpha, \beta):=\frac{\epsilon_{\alpha}+\epsilon_{\beta}}{2}$, where, $\epsilon_{\alpha} \in \mathbb{Q}^{\mathcal{P}}(\alpha \in \mathcal{P})$ has $\alpha$-coordinate equal to 1 and all other coordinates equal to 0 . This gives a (fine) grading of $A$ by the semigroup $\operatorname{im}(\mathbf{w})$. Let $\operatorname{Ch}(\mathcal{D})$ be the set of all chains in $\mathcal{D}$. Since the standard monomials (those supported on a chain) form a $\mathbb{C}$-basis for $A$, the Hilbert series with respect to this fine grading is

$$
H_{A}(r)=\sum_{c \in \operatorname{Ch}(\mathcal{D})} \sum_{\substack{a \in \operatorname{im}(\mathbf{w}) \\ \operatorname{supp}(a)=c}} r^{a}
$$


where $r:=\left(r_{\alpha} \mid \alpha \in \mathcal{P}\right), a=\left(a_{\alpha} \mid \alpha \in \mathcal{P}\right)$, and $r^{a}=\prod_{\alpha \in \mathcal{P}} r_{\alpha}^{a_{\alpha}}$. Note that elements of $\operatorname{im}(\mathbf{w})$ correspond to certain monomials with rational exponents (supported on $\mathcal{P}$ ). For example, $(\alpha, \beta) \in \mathcal{D}$ corresponds to $\sqrt{r_{\alpha} r_{\beta}}$. Setting all $r_{\alpha}=r$, we obtain the usual (coarse) Hilbert series, defined with respect to the usual $\mathbb{Z}$-grading on $A$ by degree.

Example 2.47. Consider the doset $\mathcal{D}:=\{\alpha,(\alpha, \beta), \beta\}$ on the two element poset $\{\alpha<\beta\}$. The elements of $\operatorname{Ch}(\mathcal{D})$ are shown in Figure 4 .

$$
\operatorname{Ch}(\mathcal{D})=\{\emptyset,\{\alpha\},\{\beta\},\{(\alpha, \beta)\},\{\alpha,(\alpha, \beta)\},\{(\alpha, \beta), \beta\},\{\alpha, \beta\},\{\alpha,(\alpha, \beta), \beta\}\} .
$$

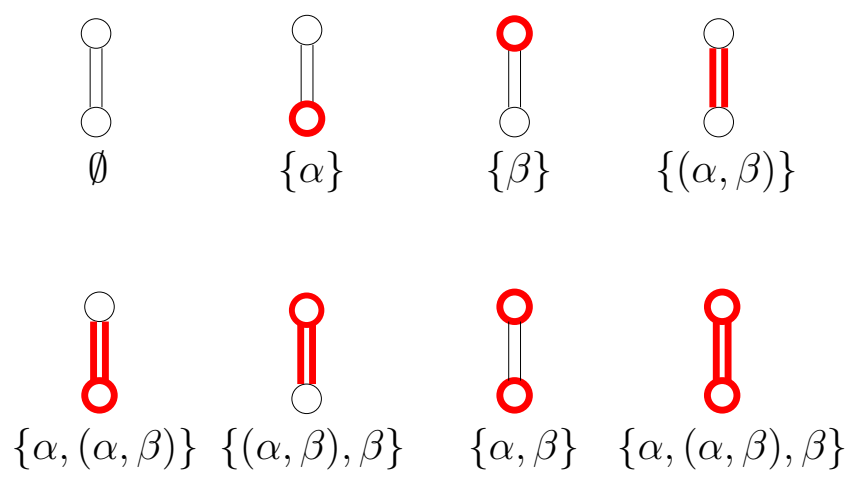

Fig. 4. The set $\operatorname{Ch}(\mathcal{D})$ of chains in $\mathcal{D}$. 
We have

$$
\begin{aligned}
H_{A}(r) & =1+\frac{r_{\alpha}}{1-r_{\alpha}}+\frac{r_{\beta}}{1-r_{\beta}}+\sqrt{r_{\alpha} r_{\beta}}+\frac{r_{\alpha}^{3 / 2} r_{\beta}^{1 / 2}}{1-r_{\alpha}} \\
& +\frac{r_{\alpha}^{1 / 2} r_{\beta}^{3 / 2}}{\left(1-r_{\beta}\right)}+\frac{r_{\alpha} r_{\beta}}{\left(1-r_{\alpha}\right)\left(1-r_{\beta}\right)}+\frac{r_{\alpha}^{3 / 2} r_{\beta}^{3 / 2}}{\left(1-r_{\alpha}\right)\left(1-r_{\beta}\right)}
\end{aligned}
$$

Setting $r=r_{\alpha}=r_{\beta}$, we obtain the Hilbert series with respect to the usual $\mathbb{Z}$-grading of $\mathbb{C}[\mathcal{D}]$.

$$
\begin{aligned}
h_{A}(r) & =1+\frac{2 r}{1-r}+r+\frac{2 r^{2}}{1-r}+\frac{r^{3}+r^{2}}{(1-r)^{2}} \\
& =\frac{r+1}{(1-r)^{2}} \\
& =1+\sum_{i=1}^{\infty}(2 i+1) r^{i} .
\end{aligned}
$$

We see that $h_{A}(i)=2 i+1, \operatorname{dim}(\operatorname{Proj} A)=1$, and $\operatorname{deg}(\operatorname{Proj} A)=2$.

Remark 2.48. The Lagrangian Grassmannian LG(2) is an algebra with straightening law on the five element doset obtained by adding two elements $\hat{0}<\alpha$ and $\hat{1}>\beta$ to the doset $\mathcal{D}$ in Example 2.47. A similar computation shows that the degree of LG(2) is also 2. Thus we confirm our stated answer to Question 1.5. Theorem 3.14 allows us to carry out such degree computations for the Drinfel'd Lagrangian Grassmannian; this gives a new derivation of numbers computed in quantum cohomology.

Fix a chain $\left\{\alpha_{1}, \ldots, \alpha_{u},\left(\beta_{11}, \beta_{12}\right), \ldots,\left(\beta_{v 1}, \beta_{v 2}\right)\right\} \subset \mathcal{D}$ (not necessarily written in order), let $t_{i}$ be the formal variable corresponding to $\alpha_{i}(i=1, \cdots, u)$, and let $s_{j k}$ correspond to $\beta_{j k}(j=1, \ldots, v, k=1,2)$. The variables $r$ and $s$ are not necessarily disjoint; in the example above, the chain $\{\alpha,(\alpha, \beta)\}$ has $r_{1}=s_{11}$. We have

$$
\sum_{\substack{a \in \operatorname{im}(\mathbf{w}) \\ \operatorname{supp}(a)=c}} r^{a}=\prod_{i=1}^{u} \frac{r_{i}}{1-r_{i}} \cdot \prod_{j=1}^{v} \sqrt{s_{j 1} s_{j 2}}
$$


Recall that we may identify $\mathcal{P}$ with the diagonal $\Delta_{\mathcal{P}} \subset \mathcal{D} \subset \mathcal{P} \times \mathcal{P}$. Letting $c_{u}^{v}$ denote the number of chains consisting of $u$ elements of $\mathcal{P}$ and $v$ elements of $\mathcal{D} \backslash \mathcal{P}$, we have

$$
\begin{aligned}
\mathrm{HS}_{A}(r) & =\sum_{u=0}^{p+1} \sum_{v=0}^{d-p} c_{u}^{v} \frac{r^{u+v}}{(1-r)^{u}} \\
& =\sum_{u=0}^{p+1} \sum_{v=0}^{d-p} c_{u}^{v} r^{u+v}\left(\sum_{k=0}^{\infty} r^{k}\right)^{u} \\
& =\sum_{v=0}^{d-p} c_{0}^{v} r^{v}+\sum_{\ell=0}^{\infty} \sum_{u=1}^{p+1} \sum_{v=0}^{d-p} c_{u}^{v}\left(\begin{array}{c}
u+\ell-1 \\
u-1
\end{array}\right) r^{u+v+\ell}
\end{aligned}
$$

When $w>d-p$, the coefficient of $r^{w}$ agrees with the Hilbert polynomial:

$$
\mathrm{HP}_{A}(w)=\sum_{u=1}^{p+1} \sum_{v=0}^{d-p} c_{u}^{v}\left(\begin{array}{c}
w-v-1 \\
u-1
\end{array}\right)
$$

In particular, the dimension of $\operatorname{Proj} A$ is $p$, since this is the largest possible value of $u-1=\operatorname{deg}_{w}\left(\begin{array}{c}w-v-1 \\ u-1\end{array}\right)$. The leading monomial of $H P_{A}(w)$ is

$$
\sum_{v=0}^{d-p} c_{p+1}^{v}\left(\begin{array}{c}
w-v-1 \\
p-1
\end{array}\right)
$$

By our assumption that the maximal chains in $\mathcal{P}$ (respectively, $\mathcal{D}$ ) have the same length, we have $c_{p+1}^{v}=\left(\begin{array}{c}d-p \\ v\end{array}\right) c_{p+1}^{0}$, so that the leading coefficient of $H P_{A}(w)$ is

$$
\frac{c_{p+1}^{0}}{(p-1) !} \sum_{v=0}^{d-p}\left(\begin{array}{c}
d-p \\
v
\end{array}\right)=\frac{2^{d-p} c_{p+1}^{0}}{(p-1) !}
$$

from which we deduce the degree of $\operatorname{Proj} A$.

Theorem 2.49. $\operatorname{deg}(\operatorname{Proj} A)=2^{d-p} c_{p}^{0}$.

Example 2.50. Let $A$ be an algebra with straightening law on the $\operatorname{doset} \mathcal{D}$ shown 
in Figure 5. We have rk $\mathcal{P}=2$, rk $\mathcal{D}=3$, and

$$
\begin{aligned}
\operatorname{Ch}(\mathcal{D})= & \{\emptyset,\{\alpha\},\{\beta\},\{\gamma\},\{\delta\},\{(\alpha, \gamma)\},\{(\beta, \delta)\}, \\
& \{\alpha, \gamma\},\{\alpha, \beta\},\{\alpha, \delta\},\{\beta, \delta\},\{\gamma, \delta\},\{\alpha,(\alpha, \gamma)\}, \\
& \{\alpha,(\beta, \delta)\},\{(\alpha, \gamma), \gamma\},\{(\alpha, \gamma), \delta\},\{\beta,(\beta, \delta)\}, \\
& \{(\beta, \delta), \delta\},\{\alpha, \beta, \delta\},\{\alpha, \gamma, \delta\},\{\alpha,(\alpha, \gamma), \gamma\} \\
& \{\alpha,(\alpha, \gamma), \delta\},\{\alpha, \beta,(\beta, \delta)\},\{\alpha,(\beta, \delta), \delta\}, \\
& \{\beta,(\beta, \delta), \delta\},\{\alpha,(\alpha, \gamma), \gamma, \delta\},\{\alpha, \beta,(\beta, \delta), \delta\}\}
\end{aligned}
$$

and the values of $c_{u}^{v}$ are given by the following matrix, whose entry in row $i$ and column $j$ is $c_{j-1}^{i-1}$ :

$$
\left(\begin{array}{llll}
1 & 4 & 5 & 2 \\
2 & 6 & 5 & 2
\end{array}\right)
$$

The Hilbert polynomial is therefore:

$$
\begin{aligned}
\mathrm{HP}_{A}(w) & =4\left(\begin{array}{c}
w-1 \\
0
\end{array}\right)+5\left(\begin{array}{c}
w-1 \\
1
\end{array}\right)+2\left(\begin{array}{c}
w-1 \\
2
\end{array}\right) \\
& +6\left(\begin{array}{c}
w-2 \\
0
\end{array}\right)+5\left(\begin{array}{c}
w-2 \\
1
\end{array}\right)+2\left(\begin{array}{c}
w-2 \\
2
\end{array}\right) \\
& =2 w^{2}+2 w+3=4 \frac{w^{2}}{2 !}+2 w+3
\end{aligned}
$$

In particular, $\operatorname{deg}(\operatorname{Proj} A)=4$.

Theorem 3.14 will allow us to compute intersection numbers in quantum cohomology using Theorem 2.49, as illustrated in Example 2.50. The appropriate doset is described in Subsection 2. We will show that the Drinfel'd Lagrangian Grassmannian is a algebra with straightening law on this doset. 


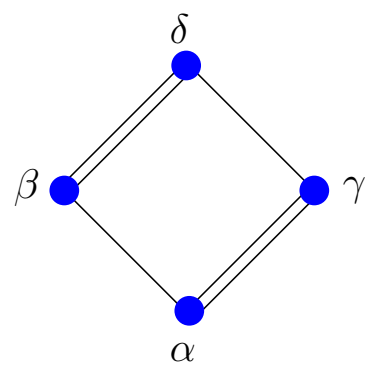

Fig. 5. The doset of Example 2.50

2. The doset of admissible pairs

We define the doset of admissible pairs on the poset $\mathcal{P}_{d, n}$. Let us first consider an example.

Example 2.51. Consider the poset

$$
\mathcal{P}_{2,4}:=\left\{\alpha^{(a)} \in\left(\begin{array}{c}
\langle 4\rangle \\
4
\end{array}\right)_{2} \mid i \in \alpha \Longleftrightarrow \bar{\imath} \notin \alpha\right\}
$$

of admissible elements of $\left(\begin{array}{c}\langle 4\rangle \\ 4\end{array}\right)_{2}$. The set $\mathcal{D}_{2,4}$ of elements $(\alpha, \beta)^{(a)} \in \mathcal{O}_{\mathcal{P}_{2,4}}$ such that $\alpha$ and $\beta$ have the same number of negative elements is a doset on $\mathcal{P}_{2,4}$. The Hasse diagram for $\mathcal{D}_{2,4}$ is shown in Figure 6 .

To each $(\alpha, \beta)^{(a)} \in \mathcal{P}_{2,4}$, we have the Plücker coordinate $p_{(\alpha, \beta)}^{(a)}:=s^{a} t^{b} \otimes p_{(\alpha, \beta)}$ in $S^{d} \mathbb{C}^{2} \otimes \mathrm{L}\left(\omega_{n}\right)$, where $S^{d} \mathbb{C}^{2}$ is the space of homogeneous polynomials of degree $d$ in two variables. 


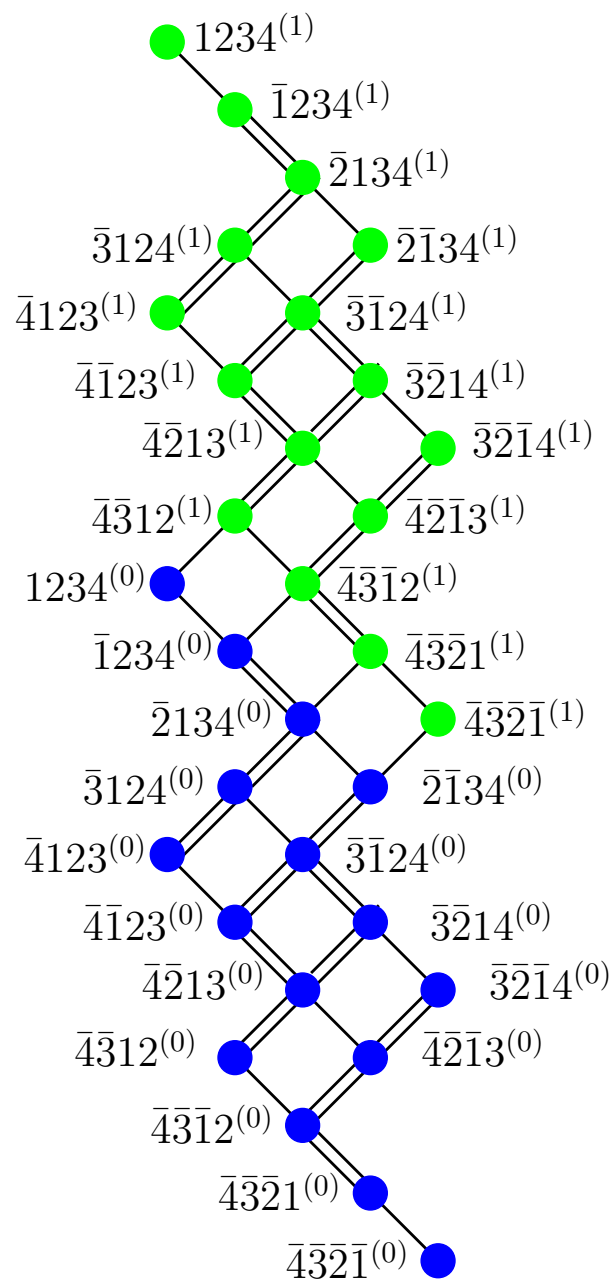

Fig. 6. The doset $\mathcal{D}_{2,4}$.

Let $\left(\begin{array}{c}\langle n\rangle \\ n\end{array}\right)_{d} \cong\left(\begin{array}{c}{[2 n]} \\ n\end{array}\right)_{d}$ be the poset associated to the Drinfel'd Grassmannian $\mathcal{Q}_{d}(n, 2 n)$ 
from the introduction, and let $\mathcal{P}_{d, n}$ be the subposet consisting of the elements $\alpha^{(a)}$ such that $\alpha^{t}=\alpha$. There are three types of covers in $\mathcal{P}_{d, n}$.

1. $\alpha^{(a)}<\beta^{(a)}$, where $\alpha$ and $\beta$ have the same number of negative elements. For example, $\overline{4} \overline{2} 13^{(a)}<\overline{4} \overline{1} 23^{(a)} \in \mathcal{P}_{d, 4}$ for any non-negative integers $a \leq d$.

2. $\alpha^{(a)}<\beta^{(a)}$, where the number of negative elements in $\beta$ is one less than the number of negative elements of $\alpha$. For example, $\overline{4} \overline{1} 23^{(a)}<\overline{4} 123^{(a)} \in \mathcal{P}_{d, 4}$ for any non-negative integers $a \leq d$.

3. $\alpha^{(a)}<\beta^{(a+1)}$, where the number of negative elements of $\beta$ is one more than the number of negative elements of $\alpha, \bar{n} \in \beta$, and $n \in \alpha$. For example, $\overline{3} \overline{2} 14^{(a)}<$ $\overline{4} \overline{3} \overline{2} 1^{(a+1)}$ for any non-negative integers $a \leq d$.

The first two types are exactly those appearing in the classical Bruhat order on $\mathcal{P}_{0, n}$. It follows that $\mathcal{P}_{d, n}$ is a union of levels $\mathcal{P}_{d, n}^{(a)}$, each isomorphic to the Bruhat order, with order relations between levels imposed by covers of the type (3) above. We define the doset $\mathcal{D}_{d, n}$ of admissible pairs in $\mathcal{P}_{d, n}$.

Definition 2.52. A pair $\left(\alpha^{(a)}<\beta^{(a)}\right)$ is admissible if there exists a saturated chain $\alpha=\alpha_{0}<\alpha_{1}<\cdots<\alpha_{s}=\beta$, where each $\alpha_{i}<\alpha_{i+1}$ is a cover of type (1).

We denote the set of admissible pairs by $\mathcal{D}_{d, n}$. Observe that $\left(\alpha^{(a)}<\beta^{(b)}\right)$ is never admissible if $a<b$.

Proposition 2.53. The set $\mathcal{D}_{d, n} \subset \mathcal{P}_{d, n} \times \mathcal{P}_{d, n}$ is a doset on $\mathcal{P}_{d, n}$. The poset $\mathcal{P}_{d, n}$ is a distributive lattice.

Proof. To show that $\mathcal{D}_{d, n}$ is a doset, one may simply apply the proof in the case $d=0$ (see [9]) to the subposet consisting of all the elements of the form $(\alpha, \beta)^{(a)}$, for each $a=0, \ldots, d$ to show that $\mathcal{D}_{d, n}$ is a doset on $\mathcal{P}_{d, n}$. 
To prove that $\mathcal{P}_{d, n}$ is a distributive lattice, we realize the meet and join as the intersection and union of certain sets (ordered by inclusion) generalizing the usual notion of a partition. Consider the union of of $n$ shifted $n \times n$ squares in $\mathbb{Z}^{2}$.

$$
S_{d, n}:=\bigcup_{a=0}^{d}\{(i-a, j-a) \mid 0 \leq i, j \leq n\}
$$

To $\alpha^{(a)} \in \mathcal{P}_{d, n}$, we associate the subset of $S_{d, n}$ defined by shifting the (open) squares in $\alpha$ by $(-a,-a)$, and adding the boxes obtained by translating a box of $\alpha$ by a vector $\left(v_{1}, v_{2}\right)$ with $v_{1}, v_{2} \leq 0$ and the points $(-i,-i)$ for $i=0, \ldots, a$. See Figure 7 for an example.

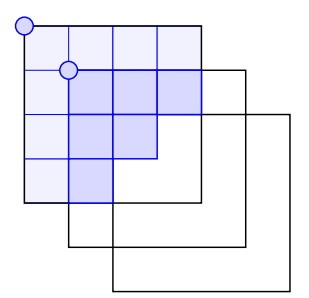

Fig. 7. The subset of $S_{2,4}$ associated to $\overline{4} \overline{2} 13^{(1)} \in \mathcal{P}_{2,4}$.

It is straightforward to check that the (symmetric) subsets obtained in this way form a distributive lattice (ordered by containment) isomorphic to $\mathcal{P}_{d, n}$. 


\section{CHAPTER III}

\section{RESULTS}

A. Algebras with straightening law

Fixing a projective variety $X \subset \mathbb{P}^{n}$, there are many homogeneous ideals $I \subset \mathbb{C}\left[x_{0}, \ldots, x_{n}\right]$ such that $\mathcal{V}(I)=X$. However, fixing a projective variety $X \subset \mathbb{P}^{n}$, there exists a unique saturated radical ideal $I$ such that $\mathcal{V}(I)=X$. Under mild hypotheses, any ideal generated by straightening relations on a doset is saturated and radical.

Theorem 3.1. Let $\mathcal{D}$ be a doset whose underlying poset has a unique minimal element $\alpha_{0}$, and let $A:=\mathbb{C}[\mathcal{D}] / J$ be an algebra with straightening law on $\mathcal{D}$, where $J$ is the ideal generated by the straightening relations. Then $J$ is saturated.

Proof. Let $f=\sum_{i=1}^{k} a_{i} m_{i} \notin J$ be a non-trivial linear combination of (distinct) standard monomials (i.e., for each $i$, the support of $m_{i}$ is a chain in $\mathcal{D}$ ). For each $N \in \mathbb{N}, p_{\alpha_{0}}^{N} f=\sum_{i=1}^{k} a_{i} p_{\alpha_{0}}^{N} m_{i}$ is a linear combination of standard monomials, since supp $m_{i} \cup\left\{\alpha_{0}\right\}$ is a chain for each $i \in[k]$. It is non-trivial since $p_{\alpha_{0}}^{N} m_{i}=p_{\alpha_{0}}^{N} m_{j}$ implies $i=j$. Thus $p_{\alpha_{0}}^{N} f \notin J$ for all $n \in \mathbb{N}$, so that $f \notin$ sat $J$.

Theorem 3.2. An algebra with straightening law on a doset is reduced.

Proof. Let $A$ be an algebra with straightening law. For $f \in A$ and $\alpha \in \mathcal{P}$, denote by $f_{\alpha}$ the restriction of $f$ to the dual Schubert variety $X^{\alpha}$.

We will show by induction on the poset $\mathcal{P}$ and on $n \in \mathbb{N}$ that $f_{\alpha}^{n}=0$ implies $f_{\alpha}=0$.

Let $f \in A$ such that $f_{\alpha}^{n}=0$ for some $n \in \mathbb{N}$. Since $f_{\beta}^{n}=0$ for all $\beta \geq \alpha, f_{\beta}=0$ by induction. It follows that $f_{\alpha}$ is supported on monomials on $X^{\alpha}$ which vanish on 
$X^{\beta}$ for all $\beta \geq \alpha$. That is,

$$
f_{\alpha}=\sum_{i=1}^{m} c_{i} p_{\alpha}^{e_{i}} p_{\left(\alpha, \beta_{1, i}\right)} \cdots p_{\left(\alpha, \beta_{\ell_{i}, i}\right)} .
$$

For the right hand side of (3.3) to be standard, we must have $\ell_{i}=1$ for all $i=1, \ldots m$. Also, homogeneity implies that $e:=e_{1}=\cdots=e_{m}$ for $i=1, \ldots m$. Thus, if we set $\beta_{1, i}:=\beta_{i}$, then $f_{\alpha}$ has the form

$$
f_{\alpha}=p_{\alpha}^{e} \sum_{i=1}^{m} c_{i} p_{\left(\alpha, \beta_{i}\right)}
$$

By induction on $n$ we may assume that $f_{\alpha}^{2}=0\left(\right.$ since $\left.\left(f_{\alpha}^{2}\right)^{\left\lceil\frac{n}{2}\right\rceil}=0\right)$.

Choose a linear extension of $\mathcal{D}$ as follows. Begin with a linear extension of $\mathcal{P} \subset \mathcal{D}$. For incomparable elements $(\alpha, \beta),(\gamma, \delta)$ of $\mathcal{D}$, set $(\alpha, \beta) \leq(\gamma, \delta)$ if $\beta<\delta$ or $\beta=\delta$ and $\alpha \leq \gamma$. With respect to the resulting linear ordering of the variables, take the lexicographic term order on monomials in $A$.

For an element $g \in A$, denote by $\operatorname{lt}(g)$ (respectively, $\operatorname{lm}(g)$ ) the lead term (respectively, lead monomial) of $g$. Reordering the terms in (3.4) if necessary, we may assume that $\operatorname{lt}\left(f_{\alpha}\right)=c_{1} p_{\alpha}^{e} p_{\left(\alpha, \beta_{1}\right)}$.

Writing $f_{\alpha}^{2}$ as a linear combination of standard monomials (by first expanding the square of the right hand side of (3.4) and then applying the straightening relations), we see that $\operatorname{lt}\left(f_{\alpha}^{2}\right)= \pm c_{1}^{2} p_{\alpha}^{2 e+1} p_{\beta_{1}}$. This follows from our choice of term order and the fourth condition in Definition 2.45.

We claim that $\operatorname{lt}\left(f_{\alpha}^{2}\right)$ cannot be canceled in the expression for $f_{\alpha}^{2}$ as a sum of standard monomials. Indeed, suppose there are $i, j \in[m]$ such that $\operatorname{lm}\left(\left(p_{\alpha}^{e} p_{\left(\alpha, \beta_{i}\right)}\right)\right.$. $\left.\left(p_{\alpha}^{e} p_{\left(\alpha, \beta_{j}\right)}\right)\right)=p_{\alpha}^{2 e+1} p_{\beta_{1}}$. Then by the straightening relations, $\beta_{1} \leq \beta_{i}, \beta_{j}$. But $\beta_{1} \nless \beta_{i}$ since $\operatorname{lm}\left(f_{\alpha}\right)=p_{\alpha}^{e} p_{\left(\alpha, \beta_{1}\right)}$. For the same reasons, $\beta_{1} \nless \beta_{j}$. Therefore $\beta_{i}=\beta_{j}=\beta_{1}$, so $c_{1} p_{\alpha}^{e} p_{\left(\alpha, \beta_{1}\right)}$ is the only term of $f_{\alpha}$ contributing to the monomial $p_{\alpha}^{2 e+1} p_{\beta_{1}}$ in $f_{\alpha}^{2}$. 


\section{B. Drinfel'd flag varieties}

\section{A basis for $S_{d} \mathbb{C}^{2} \otimes \mathrm{L}\left(\omega_{n}\right)^{*}$}

Recall that the Drinfel'd Lagrangian Grassmannian embeds in $\mathbb{P}\left(S_{d} \mathbb{C}^{2} \otimes \mathrm{L}\left(\omega_{n}\right)\right)$. We describe bases for the representation $\mathrm{L}\left(\omega_{n}\right)$ and its dual $\mathrm{L}\left(\omega_{n}\right)^{*}$.

For $\alpha \in\left(\begin{array}{c}\langle n\rangle \\ n\end{array}\right)$ and positive integers, set $v_{\alpha}^{(a)}:=s^{a} t^{d-a} \otimes e_{\alpha_{1}} \wedge \cdots \wedge e_{\alpha_{n}} \in S_{d} \mathbb{C}^{2} \otimes$ $\bigwedge^{n} \mathbb{C}^{2 n}$, and let $p_{\alpha}^{(a)}:=s^{a} t^{d-a} \otimes e_{\alpha_{1}}^{*} \wedge \cdots \wedge e_{\alpha_{n}}^{*} \in S_{d} \mathbb{C}^{2} \otimes \bigwedge^{n} \mathbb{C}^{2 n^{*}}$ be the Plücker coordinate indexed by $\alpha^{(a)} \in \mathcal{D}_{d, n}$.

The representation $\mathrm{L}\left(\omega_{n}\right)^{*}$ is the quotient of $\bigwedge^{n} \mathbb{C}^{2 n^{*}}$ by the linear subspace $L_{n}=\Omega \wedge \bigwedge^{n-2} \mathbb{C}^{2 n^{*}}$ described in Proposition 2.31. Thus $S_{d} \mathbb{C}^{2} \otimes \mathrm{L}\left(\omega_{n}\right)^{*}$ is the quotient of $S_{d} \mathbb{C}^{2} \otimes \bigwedge^{n} \mathbb{C}^{2 n^{*}}$ by the linear subspace:

$$
L_{d, n}:=S_{d} \mathbb{C}^{2} \otimes L_{n}
$$

Note that $L_{d, n}$ is spanned by linear forms

$$
\ell_{\alpha}^{(a)}:=s^{a} t^{d-a} \otimes \sum_{i \mid\{\bar{i}, i\} \cap \alpha=\emptyset} e_{\bar{\imath}}^{*} \wedge e_{i}^{*} \wedge e_{\alpha_{1}}^{*} \wedge \cdots \wedge e_{\alpha_{n-2}}^{*}
$$

for $\alpha \in\left(\begin{array}{c}\langle n\rangle \\ n-2\end{array}\right)$ and $a=0, \ldots, d$. The linear form (3.5) is simply $s^{a} t^{d-a}$ tensored with a linear form generating $L_{n}$ (Proposition 2.31). Each term in the linear form (3.5) is a Plücker coordinate indexed by a sequence $\left(\alpha_{1}<\cdots<\bar{\imath}<\cdots<i<\cdots<\alpha_{n-2}\right)^{(a)} \in$ $\left(\begin{array}{c}\langle n\rangle \\ n\end{array}\right)_{d}$, for some $i \in[n]$.

Let $T^{\prime} \subset \mathrm{SL}_{2}(\mathbb{C})$ and $T \subset \mathrm{Sp}_{2 n}(\mathbb{C})$ be maximal tori. The maximal torus $T^{\prime}$ is one dimensional, so that its Lie algebra $\mathfrak{t}^{\prime}$ has basis consisting of a single element $H \in \mathfrak{t}^{\prime}$. Let $h_{i}:=E_{i i}-E_{\overline{\imath \imath}}$ for $i \in\langle n\rangle$, and recall our convention that $\overline{\bar{\imath}}=i$, so that $h_{\bar{\imath}}=-h_{i}$. The Lie algebra $\mathfrak{t}$ of $T \subset \operatorname{Sp}_{2 n}(\mathbb{C})$ has a basis $\left\{h_{i} \mid i \in[n]\right\}$. The weights of the maximal torus $T^{\prime} \times T \subset \mathrm{SL}_{2}(\mathbb{C}) \times \operatorname{Sp}_{2 n}(\mathbb{C})$ are elements of $\mathfrak{t}^{\prime *} \oplus \mathfrak{t}^{*}$. Each Plücker coordinate $p_{\alpha}^{(a)} \in S_{d} \mathbb{C}^{2} \otimes\left(\bigwedge^{n} \mathbb{C}^{2 n}\right)^{*}$ is a weight vector of weight $(d-2 a) H^{*}+\sum_{i \mid \bar{\alpha}_{i} \notin \alpha} h_{\alpha_{i}}^{*}$. 
Each linear form (3.5) lies in a unique weight space, so we may restrict our attention to one of them. Note that the linear form $\ell_{\alpha}^{(a)}$ is nearly identical to $\ell_{\alpha}^{(b)}$; one simply changes the superscript of each Plücker coordinate from $(a)$ to $(b)$. We may therefore assume $a=0$ and write $\ell_{\alpha}$ for $\ell_{\alpha}^{(0)}$ and $p_{\alpha}$ for $p_{\alpha}^{(0)}$.

For each $\alpha \in\left(\begin{array}{c}\langle n\rangle \\ n-2\end{array}\right)$, we have an element $\ell_{\alpha}=\Omega \wedge p_{\alpha} \in L_{n}$. This is a weight vector of weight $\omega_{\alpha}:=h_{\alpha_{1}}^{*}+\cdots+h_{\alpha_{k}}^{*} \in \mathfrak{t}^{*}$. Set $\widetilde{\alpha}:=\{i \in \alpha \mid \bar{\imath} \notin \alpha\}$ and observe that $\omega_{\widetilde{\alpha}}=\omega_{\alpha}$. The elements $\alpha \in\left(\begin{array}{c}\langle n\rangle \\ n-2\end{array}\right)$ such that $\ell_{\alpha} \in\left(L_{n}\right)_{\omega}$ are those satisfying $\omega_{\alpha}=\omega$. That is, $\left(L_{n}\right)_{\omega}=\left\langle\Omega \wedge p_{\alpha} \mid \omega_{\alpha}=\omega\right\rangle$.

The shape of the linear form $\ell_{\alpha}$ is determined by the number of pairs $\{\bar{\imath}, i\} \subset \alpha$, i.e., the cardinality of $\alpha \backslash \widetilde{\alpha}$; it is the same, up to multiplication of some variables by -1 , as the linear form $\ell_{\alpha \backslash \widetilde{\alpha}}=\Omega \wedge p_{\alpha \backslash \widetilde{\alpha}} \in L_{n-2|\widetilde{\alpha}|}$, of weight $\omega_{\alpha \backslash \widetilde{\alpha}}$. It follows that the generators of $\left(L_{n}\right)_{\omega_{\alpha}}$ have the same form as those of $\left(L_{n-2|\widetilde{\alpha}|}\right)_{\omega_{\alpha}}$, up to some signs arising from sorting the elements of $\alpha$ and the indices appearing in $\Omega$. Since these signs do not affect linear independence, it suffices to find a basis for $\left(L_{n}\right)_{0}$; it is then straightforward to obtain a basis for $\left(L_{n}\right)_{\omega_{\alpha}}$. We thus assume that the weight space in question is $\left(L_{n}\right)_{0}$. This implies that $n$ is even; set $2 m:=n$.

Example 3.6. We consider linear forms which $\operatorname{span}\left(L_{6}\right)_{h_{1}^{*}+h_{3}^{*}}$. Let $m=3($ so $n=6)$ and $\omega=h_{1}^{*}+h_{3}^{*}$. If $\alpha=\overline{6} 136$, then $\widetilde{\alpha}=13$ and $\omega_{\alpha}=\omega$. We have $\ell_{\alpha}=p_{\overline{6} \overline{5} 1356}+$ $p_{\overline{6} \overline{4} 1346}-p_{\overline{6} \overline{2} 1236}$. The equations for the weight space $\left(L_{n}\right)_{\omega}$ are

$$
\begin{aligned}
& \ell_{\overline{6} 136}=p_{\overline{6} \overline{\overline{5} 1356}}+p_{\overline{6} \overline{4} 1346}-p_{\overline{6} \overline{2} 1236} \\
& \ell_{\overline{5} 135}=p_{\overline{6} \overline{\overline{5}} 1356}+p_{\overline{5} \overline{4} 1345}-p_{\overline{5} \overline{2} 1235} \\
& \ell_{\overline{4} 134}=p_{\overline{6} \overline{4} 1346}+p_{\overline{5} \overline{4} 1345}-p_{\overline{4} \overline{2} 1234} \\
& \ell_{\overline{2} 123}=p_{\overline{6} \overline{2} 1236}+p_{\overline{5} \overline{2} 1235}+p_{\overline{4} \overline{2} 1234}
\end{aligned}
$$

We can obtain the linear forms which span $\left(L_{4}\right)_{0}$ (see Example 2.39) by first removing 
every occurrence of 1 and 3 in the subscripts above and then flattening the remaining indices. That is, we apply the following replacement (and similarly for the negative indices): $6 \mapsto 4,5 \mapsto 3,4 \mapsto 2$, and $2 \mapsto 1$. We then replace a variable by its negative if 2 appears in its index; this is to keep track of the sign of the permutation sorting the sequence $\left(\bar{\imath}, i, \alpha_{1}, \ldots, \alpha_{n-2}\right)$ in each term of $\ell_{\alpha}$ (see Equation 3.5). To see why this is necessary, observe that the forms (3.5) are just $\Omega \wedge e_{\alpha_{1}}^{*} \wedge \cdots \wedge e_{\alpha_{n-2}}^{*}$.

From Proposition 2.31, it follows that the map

$$
\left(\bigwedge^{2 m} \mathbb{C}^{4 m}\right)_{0} \longrightarrow\left(\bigwedge^{2 m-2} \mathbb{C}^{4 m}\right)_{0}
$$

given by contraction with the form $\Omega \in \bigwedge^{2} \mathbb{C}^{4 m}$ is surjective, with kernel $\left(\mathrm{L}\left(\omega_{2 m}\right)\right)_{0}$. Since the set $\left\{(\bar{\alpha}, \alpha) \mid \alpha \in\left(\begin{array}{c}{[2 m]} \\ k\end{array}\right)\right\}$ is a basis of $\left(\bigwedge^{2 k} \mathbb{C}^{4 m}\right)_{0}$ (for any $k \leq m$ ), we have

$$
\begin{aligned}
\operatorname{dim}\left(\mathrm{L}\left(\omega_{2 m}\right)\right)_{0} & =\operatorname{dim}\left(\bigwedge^{2 m} \mathbb{C}^{4 m}\right)_{0}-\operatorname{dim}\left(\bigwedge^{2 m-2} \mathbb{C}^{4 m}\right)_{0} \\
& =\left(\begin{array}{c}
2 m \\
m
\end{array}\right)-\left(\begin{array}{c}
2 m \\
m-2
\end{array}\right) \\
& =\frac{1}{m+1}\left(\begin{array}{c}
2 m \\
m
\end{array}\right) .
\end{aligned}
$$

This number is equal to the number of admissible pairs of weight 0 .

Lemma 3.7. $\operatorname{dim}\left(\mathrm{L}\left(\omega_{n}\right)\right)_{0}$ is the number of admissible pairs $(\alpha, \beta) \in \mathcal{D}_{n}$ of weight $\frac{\omega_{\alpha}+\omega_{\beta}}{2}=0$.

Proof. Recall that each trivial admissible pair

$$
\alpha=\left[\bar{a}_{1}, \ldots, \bar{a}_{s}, b_{1}, \ldots, b_{n-s}\right] \in \mathcal{D}_{n}
$$

indexes a weight vector, of weight $\sum_{i=1}^{n-s} h_{b_{i}}^{*}-\sum_{i=1}^{s} h_{a_{i}}^{*}$. Also, the non-trivial admissible pairs are those $(\alpha, \beta)$ for which $\alpha<\beta$ have the same number of negative elements. From this it follows that the admissible pairs of weight zero are the $(\alpha, \beta) \in \mathcal{D}_{n}$ 
such that $\beta=\left[\bar{a}_{m}, \ldots, \bar{a}_{1}, b_{1}, \ldots, b_{m}\right], \alpha=\left[\bar{b}_{m}, \ldots, \bar{b}_{1}, a_{1}, \ldots, a_{m}\right],\left\{a_{1}, \ldots, a_{m}\right\}$ and $\left\{b_{1}, \ldots, b_{m}\right\}$ are disjoint. This last condition is equivalent to $a_{i}>b_{i}$ for all $i \in[m]$. The number of such pairs is equal to the number of standard tableaux of shape $\left(\mathrm{m}^{2}\right)$ (that is, a rectangular box with 2 rows and $m$ columns) with entries in $[2 m]$. By the hook length formula [12] this number is $\frac{1}{m+1}\left(\begin{array}{c}2 m \\ m\end{array}\right)$.

The weight vectors $p_{\alpha} \in\left(\bigwedge^{n} \mathbb{C}^{4 m^{*}}\right)_{0}$ are indexed by sequences of the form

$$
\alpha=\left(\bar{\alpha}_{m}, \ldots, \bar{\alpha}_{1}, \alpha_{1}, \ldots, \alpha_{m}\right)
$$

which can be abbreviated by the positive subsequence $\alpha_{+}:=\left(\alpha_{1}, \ldots, \alpha_{m}\right) \in\left(\begin{array}{c}{[2 m]} \\ m\end{array}\right)$ without ambiguity. We take these as an indexing set for the variables appearing in the linear forms (3.5).

With this notation, the positive parts of Northeast sequences are characterized in Proposition 3.9. The proof requires the following definition.

Definition 3.8. A tableau is a partition whose boxes are filled with integers from the set $[n]$, for some $n \in \mathbb{N}$. A tableau is standard if the entries strictly increase from left to right and top to bottom.

Proposition 3.9. Let $\alpha \in\left(\begin{array}{c}(2 m\rangle \\ 2 m\end{array}\right)$ be a Northeast sequence. Then the positive part of $\alpha$ satisfies $\alpha_{+} \geq 24 \cdots(2 m) \in\left(\begin{array}{c}{[2 m]} \\ m\end{array}\right)$. In particular, no Northeast sequence contains $1 \in[2 m]$ and every Northeast sequence contains $2 m \in[2 m]$.

Proof. $\alpha_{+} \geq 24 \cdots(2 m)$ if and only if the tableau of shape $\left(m^{2}\right)$ whose first row is filled with the sequence $\left(\alpha^{t}\right)_{+}=[n] \backslash \alpha_{+}$and whose second row is filled with the $\alpha_{+}$ is standard. This is equivalent to $\alpha$ being Northeast.

It follows from Proposition 2.38 that the set $\mathcal{N E}$ of Northeast sequences indexing vectors of weight zero has cardinality equal to the dimension of the zero-weight space 
of the representation $\mathrm{L}\left(\omega_{2 m}\right)^{*}$. This weight space is the cokernel of the map

$$
\Omega \wedge \bullet:\left(\bigwedge^{2 m-2} \mathbb{C}^{4 m}\right)_{0}^{*} \longrightarrow\left(\bigwedge^{2 m} \mathbb{C}^{4 m}\right)_{0}^{*}
$$

Similarly, the weight space $\mathrm{L}\left(\omega_{2 m}\right)_{0}$ is the kernel of the dual map

$$
\Omega\lrcorner \bullet:\left(\bigwedge^{2 m} \mathbb{C}^{4 m}\right)_{0} \longrightarrow\left(\bigwedge^{2 m-2} \mathbb{C}^{4 m}\right)_{0}
$$

We fix the positive integer $m$, and consider only the positive part (in $\left(\begin{array}{c}{[2 m]} \\ m\end{array}\right)$ ) of elements of $\left(\begin{array}{c}\langle 4 m\rangle \\ 2 m\end{array}\right)$ such that the associated Plücker coordinate has weight zero. For $\alpha \in\left(\begin{array}{c}{[2 m]} \\ m\end{array}\right)$, a matching is a bijection $M: \alpha \rightarrow \alpha^{c}$. For any matching, we have an element of the kernel $\mathrm{L}\left(\omega_{2 m}\right)$ of $\left.\Omega\right\lrcorner \bullet$ : Let $H_{\alpha}$ be the set of all sequences in $\left(\begin{array}{c}{[2 m]} \\ m\end{array}\right)$ obtained by interchanging $M\left(\alpha_{i}\right)$ and $\alpha_{i}$, for $i \in I, I \subset[m]$. Elements of the set $H_{\alpha}$ are the vertices of a hypercube, whose edges connect pairs of sequences which are related by the interchange of a single element. Equivalently, a pair of sequences are connected by an edge if they share a subsequence of size $m-1$. Observe that for a subsequence $\beta \subset \alpha$ of length $m-1$, there exists a unique edge of $H_{\alpha}$ connecting two vertices with common subsequence $\beta$. Let $I \cdot \alpha$ denote the element of $H_{\alpha}$ obtained from $\alpha$ by the interchange of $M\left(\alpha_{i}\right)$ and $\alpha_{i}$ for $i \in I$. The element

$$
K_{\alpha}:=\sum_{I \subset[m]}(-1)^{|I|} v_{I \cdot \alpha}
$$

lies in $\mathrm{L}\left(\omega_{2 m}\right)$. Indeed, for each $I \subset[m]$, we have

$$
\Omega\lrcorner v_{I \cdot \alpha}=\sum_{i=1}^{m} v_{(I \cdot \alpha) \backslash\left\{(I \cdot \alpha)_{i}\right\}} .
$$

For each term $(-1)^{|I|} v_{(I \cdot \alpha) \backslash\left\{(I \cdot \alpha)_{i}\right\}}$ on the right-hand side, suppose that $j \in[m]$ is such that either $(I \cdot \alpha)_{i}=\alpha_{j}$ or $(I \cdot \alpha)_{i}=\alpha_{j}^{c}$. Set $J:=I \cup\{j\}$ if $(I \cdot \alpha)_{i}=\alpha_{j}$ and $J:=I \backslash\{j\}$ if $(I \cdot \alpha)_{i}=\alpha_{j}^{c}$. The set $J$ is the unique subset of $[m]$ such that $(I \cdot \alpha) \backslash\left\{(I \cdot \alpha)_{i}\right\}$ is in 
the support of $\Omega\lrcorner v_{J \cdot \alpha}$, with coefficient $(-1)^{|J|}=(-1)^{|I|+1}$. Hence these terms cancel, and we see that the coefficient of each $\beta \in\left(\begin{array}{c}{[2 m]} \\ m-1\end{array}\right)$ with $v_{\beta}$ in the support of $\left.\Omega\right\lrcorner K_{\alpha}$ is zero. Therefore $\Omega\lrcorner K_{\alpha}=0$. See Example 3.13 for the case $m=2$.

If $\alpha \in \mathcal{N E}$ then that there exists a descending matching, that is, $M\left(\alpha_{i}\right)<\alpha_{i}$ for all $i \in[m]$. For example, the condition that the matching $M\left(\alpha_{i}\right):=\alpha_{i}^{c}$ be descending is equivalent to the condition that $\alpha$ be Northeast. If we choose a descending matching for each $\alpha \in \mathcal{N} \mathcal{E}$, the element $K_{\alpha} \in \mathrm{L}\left(\omega_{2 m}\right)$ involves only sequences which preceed $\alpha$. It follows that the set $\mathcal{B}:=\left\{K_{\alpha} \in \mathrm{L}\left(\omega_{2 m}\right) \mid \alpha \in \mathcal{N} \mathcal{E}\right\}$ is a basis for $\mathrm{L}\left(\omega_{2 m}\right)$.

Lemma 3.10. The Plücker coordinates $p_{\alpha}$ with $\alpha \in \mathcal{N} \mathcal{E}$ are a basis for $\mathrm{L}\left(\omega_{2 m}\right)^{*}$.

Proof. Fix a basis $\mathcal{B}$ of $\mathrm{L}\left(\omega_{2 m}\right)$ obtained from descending matchings of each Northeast sequence with its dual. We can use this basis to show that the set of Plücker coordinates $p_{\alpha}$ such that $\alpha$ is Northeast is a basis for the dual $\mathrm{L}\left(\omega_{2 m}\right)^{*}$.

Suppose not. Then there exists a linear form $\ell=\sum_{\alpha \in \mathcal{N} \mathcal{E}} c_{\alpha} p_{\alpha}$ vanishing on each element of the basis $\mathcal{B}$. We show by induction on $\mathcal{N} \mathcal{E}$ that all of the coefficients $c_{\alpha}$ appearing in this form vanish.

Fix a Northeast sequence $\alpha \in \mathcal{N} \mathcal{E}$, and assume that $c_{\beta}=0$ for all Northeast $\beta<\alpha$. Since $K_{\alpha}$ involves only the basis vectors $v_{\beta}$ with $\beta \leq \alpha$, we have $\ell\left(K_{\alpha}\right)=c_{\alpha}$, hence $c_{\alpha}=0$. This completes the inductive step of the proof.

The initial step of the induction is simply the inductive step applied to the unique minimal Northeast sequence $\alpha=24 \cdots(2 m)$.

It follows that every Plücker coordinate $p_{\alpha}$ indexed by a non-Northeast sequence $\alpha$ can be written uniquely as a linear combination of Plücker coordinates indexed by Northeast sequences. We can be more precise about the form of these linear combinations. Recall that each fiber of the map $\pi_{2 m}$ contains a unique Northeast sequence. For a sequence $\alpha_{0}$, let $\alpha$ be the Northeast sequence in the same fiber as $\alpha_{0}$. 
Lemma 3.11. For each non-Northeast sequence $\alpha_{0}$, let $\ell_{\alpha_{0}}^{\prime}$ be the linear relation among the Plücker coordinates expressing $p_{\alpha_{0}}$ as a linear combination of the $p_{\beta}$ with $\beta$ Northeast. Then $p_{\alpha}$ appears in $\ell_{\alpha_{0}}^{\prime}$ with coefficient $(-1)^{|I|}$, where $\alpha=I \cdot \alpha_{0}$, and every other Northeast $\beta$ with $p_{\beta}$ in the support of $\ell_{\alpha_{0}}^{\prime}$ satisfies $\beta>\alpha$.

Proof. Let $M$ be the descending matching of $\alpha$ with $\alpha^{c}$ defined by $M\left(\alpha_{i}\right):=\alpha_{i}^{c}$. Let $K_{\alpha}$ be the kernel element obtained by the process described above. Any linear form

$$
\ell=p_{\alpha_{0}}+(-1)^{|I|+1} p_{\alpha}+\sum_{\alpha<\beta \in \mathcal{N} \mathcal{E}} c_{\beta} p_{\beta}
$$

vanishes on $K_{\alpha}$.

We extend this relation to one which vanishes on all of $\mathrm{L}\left(\omega_{n}\right)_{0}$, proceeding inductively on the poset of Northeast sequences greater than or equal to $\alpha$. Suppose that $\beta>\alpha$ is Northeast. By induction, suppose that for each Northeast sequence $\gamma$ in the interval $[\alpha, \beta]$ the coefficient $c_{\gamma}$ of $\ell$ has been determined in such a way that $\ell\left(K_{\gamma}\right)=0$

Let $S$ be the set of Northeast sequences $\gamma$ in the open interval $(\alpha, \beta)$ such that $v_{\gamma}$ appears in $K_{\beta}$. Then

$$
\ell\left(K_{\beta}\right)=\left(\sum_{\gamma \in S} c_{\gamma}\right)+c_{\beta},
$$

so setting $c_{\beta}:=-\sum_{\gamma \in S} c_{\gamma}$ implies that $\ell\left(K_{\beta}\right)=0$.

This completes the inductive part of the proof. We now have a linear form $\ell$ vanishing on $\mathrm{L}\left(\omega_{n}\right)_{0}$ which expresses $p_{\alpha_{0}}$ as a linear combination of Plücker coordinates indexed by Northeast sequences. Since such a linear form is unique, $\ell=\ell_{\alpha_{0}}^{\prime}$.

By Lemmas 3.10 and 3.11 and the argument preceding them, we have the following theorem.

Theorem 3.12. The system of linear relations $\left\{\ell_{\alpha}^{(a)}=s^{a} t^{d-a} \otimes \Omega \wedge p_{\alpha} \mid a=\right.$ 
$\left.0, \ldots, d, \alpha \in\left(\begin{array}{c}\langle n\rangle \\ n-2\end{array}\right)\right\}$ has a reduced normal form consisting of linear forms expressing each Plücker coordinate $p_{\beta}^{(b)}$ with $\beta \notin \mathcal{N E} \subset\left(\begin{array}{c}\langle n\rangle \\ n\end{array}\right)$ as a linear combination of Plücker coordinates indexed by Northeast elements of $\left(\begin{array}{c}\langle n\rangle \\ n\end{array}\right)$.

Proof. We have seen that the linear relations preserve weight spaces, and Lemmas 3.10 and 3.11 provide the required normal form on each of these. The union of the relations constitute a normal form for the linear relations generating the entire linear subspace $L_{d, n}$

Example 3.13. Consider the zero weight space $\left(\bigwedge^{4} \mathbb{C}^{8}\right)_{0}$ (so that $\left.m=2\right)$. This is spanned by the vectors $v_{\alpha}:=e_{\alpha_{1}} \wedge e_{\alpha_{2}} \wedge e_{\alpha_{3}} \wedge e_{\alpha_{4}}$ (with dual basis the Plücker coordinates $p_{\alpha}=v_{\alpha}^{*}$ ), where $\alpha \in\{\overline{4} \overline{3} 34, \overline{4} \overline{2} 24, \overline{4} \overline{1} 14, \overline{3} \overline{2} 23, \overline{3} \overline{1} 13, \overline{2} \overline{1} 12\}$. The Northeast sequences are $\overline{4} \overline{3} 34$ and $\overline{4} \overline{2} 24$. The kernel of $\Omega\lrcorner \bullet:\left(\bigwedge^{4} \mathbb{C}^{8}\right)_{0} \rightarrow\left(\bigwedge^{2} \mathbb{C}^{8}\right)_{0}$ is spanned by the vectors

$$
K_{\overline{4} \overline{2} 24}=v_{\overline{4} \overline{2} 24}-v_{\overline{4} \overline{1} 14}-v_{\overline{3} \overline{2} 23}+v_{\overline{3} \overline{1} 13}
$$

and

$$
K_{\overline{4} \overline{3} 34}=v_{\overline{4} \overline{3} 34}-v_{\overline{4} \overline{1} 14}-v_{\overline{3} \overline{2} 23}+v_{\overline{2} \overline{1} 12} .
$$

To see this concretely, we compute:

$$
\begin{aligned}
\Omega\lrcorner K_{\overline{4} \overline{2} 24} & =v_{\overline{4} 4}+v_{\overline{2} 2}-v_{\overline{4} 4}-v_{\overline{1} 1}-v_{\overline{3} 3}-v_{\overline{2} 2}+v_{\overline{3} 3}+v_{\overline{1} 1} \\
& =0
\end{aligned}
$$

and similarly $\Omega\lrcorner K_{\overline{4} \overline{3} 34}=0$. The fibers of the map $\pi_{4}:\left(\begin{array}{c}\langle 4\rangle \\ 4\end{array}\right) \rightarrow \mathcal{D}_{4}$ are

$$
\pi_{4}^{-1}(\overline{4} \overline{3} 12, \overline{2} \overline{1} 34)=\{\overline{4} \overline{3} 34, \overline{2} \overline{1} 12\}
$$

and

$$
\pi_{4}^{-1}(\overline{4} \overline{2} 13, \overline{3} \overline{1} 24)=\{\overline{4} \overline{2} 24, \overline{4} \overline{1} 14, \overline{3} \overline{2} 23, \overline{3} \overline{1} 13\}
$$


The expression for $p_{\overline{4} \overline{1} 14}$ as a linear combination of Plücker coordinates indexed by Northeast sequences is

$$
\ell_{\overline{4} \overline{1} 14}=p_{\overline{4} \overline{1} 14}+c_{\overline{4} \overline{2} 24} p_{\overline{4} \overline{2} 24}+c_{\overline{4} \overline{3} 34} p_{\overline{4} \overline{3} 34}
$$

for some $c_{\overline{4} \overline{2} 24}, c_{\overline{4} \overline{3} 34} \in \mathbb{C}$. Since $\ell_{\overline{4} \overline{1} 14}\left(K_{\overline{4} \overline{2} 24}\right)=c_{\overline{4} \overline{2} 24}-1$, we take $c_{\overline{4} \overline{2} 24}=1$. Similarly, $\ell_{\overline{4} \overline{1} 14}\left(K_{\overline{4} \overline{3} 34}\right)=c_{\overline{4} \overline{3} 34}-1$, so $c_{\overline{4} \overline{3} 34}=1$. Hence $\ell_{\overline{4} \overline{1} 14}=p_{\overline{4} \overline{1} 14}+p_{\overline{4} \overline{2} 24}+p_{\overline{4} \overline{3} 34}$, which agrees with (2.41).

\section{Proof and consequences of the straightening law}

We wish to find generators of $\left(I_{d, n}+L_{d, n}\right) \cap \mathbb{C}\left[\mathcal{D}_{d, n}\right]$ which express the quotient as an algebra with straightening law on $\mathcal{D}_{d, n}$. Such a generating set is automatically a Gröbner basis with respect to the degree reverse lexicographic term order where variables are ordered by a refinement of the doset order. We begin with a Gröbner basis $G_{I_{d, n}+L_{d, n}}$ for $I_{d, n}+L_{d, n}$ with respect to a similar term order. For $\alpha^{(a)} \in\left(\begin{array}{c}\langle n\rangle \\ n\end{array}\right)_{d}$, write $\check{\alpha}^{(a)}:=\alpha^{(a)} \vee\left(\alpha^{t}\right)^{(a)}$ and $\hat{\alpha}^{(a)}:=\alpha^{(a)} \wedge\left(\alpha^{t}\right)^{(a)}$, so that $\pi_{n}\left(\alpha^{(a)}\right)=\left(\hat{\alpha}^{(a)}, \check{\alpha}^{(a)}\right)$.

Let $<$ be a linear refinement of the partial order on $\mathcal{P}_{d, n}$ satisfying the following conditions. First, the Northeast sequence is minimal among those in a given fiber of $\pi_{n}$. This is possible since every weight space is supported on a antichain (i.e., no two elements are comparable). Second, $\alpha^{(a)}<\beta^{(b)}$ if $\left(\hat{\alpha}^{(a)}, \check{\alpha}^{(a)}\right)$ is lexicographically smaller than $\left(\hat{\beta}^{(b)}, \check{\beta}^{(b)}\right)$.

Consider the resulting degree reverse lexicographic term order. A reduced Gröbner basis $G_{d, n}$ for $I_{d, n}+L_{d, n}$ with respect to this term order will have standard monomials indexed by chains (in $\mathcal{P}_{d, n}$ ) of Northeast partitions. While every monomial supported on a chain of Northeast partitions is standard modulo $I_{d, n}$, this is not always the case modulo $I_{d, n}+L_{d, n}$. In other words, upon identifying each Northeast partition appearing in a given monomial with an element of $\mathcal{D}_{d, n}$, we do not necessarily obtain 
a monomial supported on a chain in $\mathcal{D}_{d, n}$. It is thus necessary to identify precisely which Northeast chains in $\left(\begin{array}{c}\langle n\rangle \\ n\end{array}\right)$ correspond to chains in $\mathcal{D}_{d, n}$ via the map $\pi_{n}$.

A monomial $p_{\alpha}^{(a)} p_{\beta}^{(b)}$ with $\alpha^{(a)}<\beta^{(b)},\left(\beta^{t}\right)^{(b)}$ and $\alpha^{(a)}, \beta^{(b)}$ both Northeast cannot be reduced modulo $G_{I_{d, n}}$ or $G_{L_{d, n}}$. On the other hand, if $\alpha^{(a)}<\beta^{(b)}$ (say), but $\alpha^{(a)}$ and $\left(\beta^{t}\right)^{(b)}$ are incomparable (written $\left.\alpha^{(a)} \nsim\left(\beta^{t}\right)^{(b)}\right)$ then there is a relation in $G_{I_{d, n}}$ with leading term $p_{\alpha}^{(a)} p_{\beta^{t}}^{(b)}$. It follows that the degree-two standard monomials are indexed by Northeast partitions $p_{\alpha}^{(a)} p_{\beta}^{(b)}$ with $\alpha^{(a)}<\beta^{(b)},\left(\beta^{t}\right)^{(b)}$.

Conversely, any monomial $p_{\alpha}^{(a)} p_{\beta}^{(b)}$ with $\alpha^{(a)}<\beta^{(b)},\left(\beta^{t}\right)^{(b)}$ cannot be the leading term of any element of $G_{I_{d, n}+L_{d, n}}$. To see this, observe that $G_{I_{d, n}+L_{d, n}}$ is obtained by Buchberger's algorithm applied to $G_{I_{d, n}} \cup G_{L_{d, n}}$, and we may consider only the $S$-polynomials $S(f, g)$ with $f \in G_{I_{d, n}}$ and $g \in G_{L_{d, n}}$. By Proposition 2.10, any such $S$-polynomial reduces to zero, unless $\operatorname{in}_{<} g$ divides $\operatorname{in}_{<} f$.

Let $\alpha_{0}$ be the partition such that $\operatorname{in}_{<} f=p_{\alpha_{0}}^{(a)}$ (that is, $f$ is the unique expression of $p_{\alpha_{0}}^{(a)}$ as a linear combination of Plücker coordinates indexed by Northeast partitions), and let $\alpha$ be the unique Northeast partition such that $\pi_{n}\left(\alpha_{0}\right)=\pi_{n}(\alpha)$. By the reduced normal form given in Theorem $3.12, S(f, g)$ is the obtained by replacing $p_{\alpha_{0}}^{(a)}$ with $\pm p_{\alpha}^{(a)}+\ell$, where $\ell$ is a linear combination of Plücker coordinates $p_{\gamma}^{(a)}$ with $\gamma$ Northeast and $\alpha_{+}<\gamma_{+}$. This latter condition implies that $\hat{\alpha}<\hat{\gamma}$ (also, $\check{\alpha}>\check{\gamma}$ ), and therefore $(\hat{\alpha}, \check{\alpha})$ is lexicographically smaller than $(\hat{\gamma}, \check{\gamma})$.

Hence the standard monomials with respect to the reduced Gröbner basis $G_{I_{d, n}+L_{d, n}}$ are precisely the $p_{\alpha}^{(a)} p_{\beta}^{(b)}$ with $\alpha^{(a)}<\beta^{(b)},\left(\beta^{t}\right)^{(b)}$.

Recall that elements of the doset $\mathcal{D}_{d, n}$ are pairs $(\alpha, \beta)$ of admissible (Definition 2.34) elements of $\left(\begin{array}{c}\langle n\rangle \\ n\end{array}\right)_{d}$ such that (regarded as sequences):

- $\alpha<\beta$

- $\alpha$ and $\beta$ have the same number of negative (or positive) elements 
Equivalently, regarding $\alpha$ and $\beta$ as partitions, the elements of $\mathcal{D}_{d, n}$ are pairs $(\alpha, \beta)$ of symmetric partitions such that

- $\alpha \subseteq \beta$,

- $\alpha$ and $\beta$ have the same Durfee square,

where the Durfee square of a partition $\alpha$ is the largest square subpartition $\left(p^{p}\right) \subset \alpha$ (for some $p \leq n$ ).

Theorem 3.14. $\mathbb{C}\left[\left(\begin{array}{c}\langle n\rangle \\ n\end{array}\right)_{d}\right] /\left\langle I_{d, n}+L_{d, n}\right\rangle$ is an algebra with straightening law on $\mathcal{D}_{d, n}$.

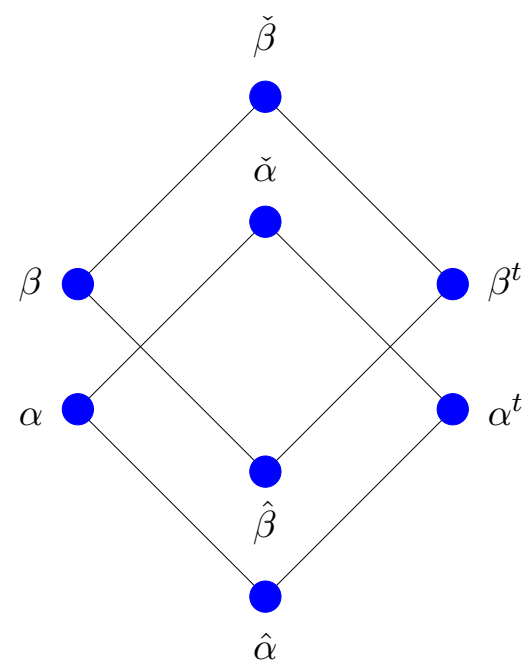

Case 1

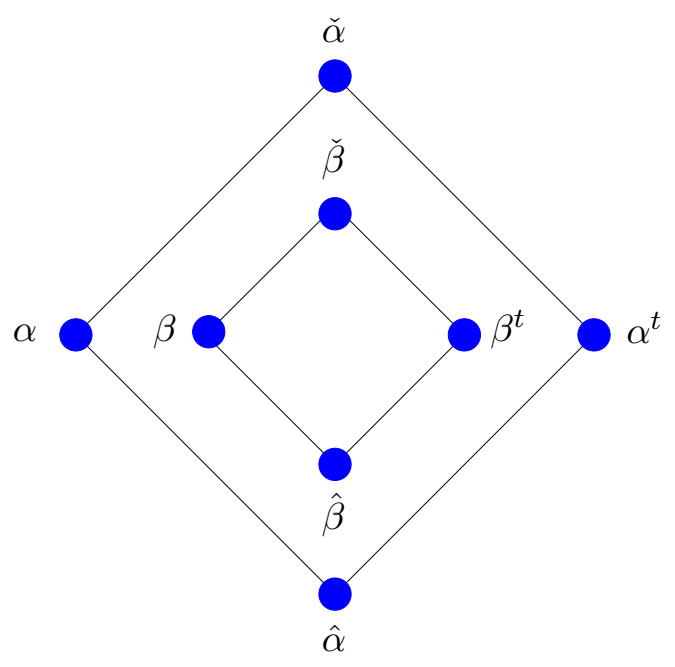

Case 2

Fig. 8. The two cases in the proof of fourth condition in Definition 2.45. 
Proof. Since standard monomials with respect to a Gröbner basis are linearly independent, the arguments above establish the first two conditions in Definition 2.45.

To establish the third condition, note that it suffices to consider the expression for a degree-2 monomial as a sum of standard monomials. For simplicity, we absorb the superscripts into our notation and write $\alpha \in\left(\begin{array}{c}\langle n\rangle \\ n\end{array}\right)_{d}$ and similarly for the corresponding Plücker coordinate. Let

$$
p_{(\hat{\alpha}, \check{\alpha})} p_{(\hat{\beta}, \check{\beta})}=\sum_{j=1}^{k} c_{j} p_{\left(\hat{\alpha}_{j}, \check{\alpha}_{j}\right)} p_{\left(\hat{\beta}_{j}, \check{\beta}_{j}\right)}
$$

be a reduced expression in $G_{I_{d, n}+L_{d, n}}$ for $p_{(\hat{\alpha}, \breve{\alpha})} p_{(\hat{\beta}, \breve{\beta})}$ as a sum of standard monomials. That is, $p_{(\hat{\alpha}, \breve{\alpha})} p_{(\hat{\beta}, \breve{\beta})}$ is non-standard and $p_{\left(\hat{\alpha}_{j}, \check{\alpha}_{j}\right)} p_{\left(\hat{\beta}_{j}, \check{\beta}_{j}\right)}$ is standard for $j=1, \ldots, k$, where $\alpha$ (respectively, $\beta$ ) be the unique Northeast partition such that $\pi_{n}(\alpha)=(\hat{\alpha}, \check{\alpha})$ (respectively, $\left.\pi_{n}(\beta)=(\hat{\beta}, \check{\beta})\right)$, and similarly for each $\alpha_{j}$ and $\beta_{j}$.

Fix $j=1, \ldots, k$. The standard monomial $p_{\left(\hat{\alpha}_{j}, \check{\alpha}_{j}\right)} p_{\left(\hat{\beta}_{j}, \check{\beta}_{j}\right)}$ is obtained by the reduction of a standard monomial $p_{\gamma} p_{\delta}$ appearing in an element of the Gröbner basis $G_{I_{d, n}}$. If $\gamma$ and $\delta$ are both Northeast, then nothing happens, i.e., $\gamma=\alpha_{j}$ and $\delta=\beta_{j}$. If $\gamma$ is not Northeast, then we rewrite $p_{\gamma}$ as a linear combination of Plücker coordinates indexed by Northeast sequences. Lemma 3.11 ensures that the leading term of the new expression is $p_{(\hat{\gamma}, \breve{\gamma})}$, and the lower order terms $p_{(\hat{\epsilon}, \breve{\epsilon})}$ satisfy $\hat{\epsilon}<\hat{\gamma}$. The lexicographic comparison in the third condition of Definition 2.45 terminates with the first Plücker coordinate. That is, if $\left(\hat{\alpha}_{j} \leq \check{\alpha}_{j} \leq \hat{\beta}_{j} \leq \check{\beta}_{j}\right)$ is lexicographically smaller than $(\hat{\alpha} \leq \check{\alpha} \leq \hat{\beta} \leq \check{\beta})$, then either $\hat{\alpha}_{j}<\hat{\alpha}$ or $\hat{\alpha}_{j}=\hat{\alpha}$ and $\hat{\alpha}_{j}<\hat{\alpha}$. Therefore the reduction process applied to $p_{\delta}$ does not affect the result, and the third condition is proven.

It remains to prove the fourth condition. Suppose that $(\hat{\alpha}, \check{\alpha})$ and $(\hat{\beta}, \check{\beta})$ are incomparable elements of $\mathcal{D}_{d, n}$ ( $\alpha$ and $\beta$ Northeast). This means that $\alpha$ is incomparable to either $\beta$ or $\beta^{t}$ (possibly both). Without loss of generality, we will deal only with the more complicated case that $\alpha$ and $\beta^{t}$ are incomparable. The hypothesis of 
the fourth condition is that the set $\{\hat{\alpha}, \check{\alpha}, \hat{\beta}, \check{\beta}\}$ forms a chain in $\left(\begin{array}{c}\langle n\rangle \\ n\end{array}\right)_{d}$. Up to interchanging the roles of $\alpha$ and $\beta$, there are two possible cases: either $\hat{\alpha}<\hat{\beta}<\check{\alpha}<\check{\beta}$ or $\hat{\alpha}<\hat{\beta}<\check{\beta}<\check{\alpha}$. We visualize each case in Figure 8 .

Suppose $\hat{\alpha}<\hat{\beta}<\check{\alpha}<\check{\beta}$. Recall that for any $\gamma_{0} \in\left(\begin{array}{c}\langle n\rangle \\ n\end{array}\right)_{d}$, with Northeast sequence $\gamma$ in the same fiber of $\pi_{n}$, the expression for the Plücker coordinate $p_{\gamma_{0}}$ as a linear combination of Plücker coordinates indexed by Northeast sequences is supported on Plücker coordinates $p_{\delta}$ such that $\delta_{+} \geq \gamma_{+}$, with equality if and only if $\delta=\gamma$, and the Plücker coordinate $p_{\gamma}$ appears with coefficient \pm 1 (Lemma 3.11).

Upon replacing each Northeast (or Southwest) partition with its associated doset element using the map $\pi_{n}$ from Chapter II, Section 1, the first two terms of straightening relation for $p_{\alpha} p_{\beta^{t}}$ are

$$
\begin{aligned}
p_{\alpha} p_{\beta^{t}}-p_{\alpha \wedge \beta^{t}} p_{\alpha \vee \beta^{t}} & =p_{\alpha} p_{\beta^{t}}-\sigma p_{\left(\left(\alpha \wedge \beta^{t}\right)^{\wedge},\left(\alpha \wedge \beta^{t}\right)^{\vee}\right)} p_{\left(\left(\alpha \vee \beta^{t}\right)^{\wedge},\left(\alpha \vee \beta^{t}\right)^{\vee}\right)}+\text { lower order terms } \\
& =\sigma_{\beta} p_{(\hat{\alpha}, \check{\alpha})} p_{(\hat{\beta}, \check{\beta})}-\sigma p_{(\hat{\alpha}, \hat{\beta})} p_{(\check{\alpha}, \check{\beta})}+\text { lower order terms, }
\end{aligned}
$$

where $\sigma= \pm 1$. The second equation is justified by the following computation in the distributive lattice $\left(\begin{array}{c}\langle n\rangle \\ n\end{array}\right)_{d}$.

$$
\begin{aligned}
\left(\alpha \wedge \beta^{t}\right) \wedge\left(\alpha^{t} \wedge \beta\right) & =\left(\alpha \wedge \alpha^{t}\right) \wedge\left(\beta \wedge \beta^{t}\right) \\
& =\hat{\alpha} \wedge \hat{\beta} \\
& =\hat{\alpha} \\
\left(\alpha \wedge \beta^{t}\right) \vee\left(\alpha^{t} \wedge \beta\right) & =\left(\alpha \vee \alpha^{t}\right) \wedge\left((\alpha \vee \beta) \wedge\left(\beta^{t} \vee \alpha^{t}\right)\right) \wedge\left(\beta^{t} \vee \beta\right) \\
& =\check{\alpha} \wedge\left(\hat{\alpha} \vee\left(\alpha \wedge \beta^{t}\right)^{\vee} \vee \hat{\beta}\right) \wedge \check{\beta} \\
& =\check{\alpha} \wedge \hat{\beta} \wedge \check{\beta} \\
& =\hat{\beta}
\end{aligned}
$$


Similarly, $\left(\alpha \vee \beta^{t}\right) \wedge\left(\alpha^{t} \vee \beta\right)=\check{\alpha}$ and $\left(\alpha \vee \beta^{t}\right) \vee\left(\alpha^{t} \vee \beta\right)=\check{\beta}$

If $\check{\alpha}<\hat{\beta}<\check{\beta}<\check{\alpha}$, then we have both $\alpha \not \beta$ and $\alpha \not \beta^{t}$. We use the relation for the incomparable pair $\alpha \not \beta^{t}$.

$$
\begin{aligned}
p_{\alpha} p_{\beta^{t}}-p_{\alpha \wedge \beta^{t}} p_{\alpha \vee \beta^{t}} & =p_{\alpha} p_{\beta^{t}}-\sigma p_{\left(\left(\alpha \wedge \beta^{t}\right)^{\wedge},\left(\alpha \wedge \beta^{t}\right)^{\vee}\right)} p_{\left(\left(\alpha \vee \beta^{t}\right)^{\wedge},\left(\alpha \vee \beta^{t}\right)^{\vee}\right)}+\text { lower order terms } \\
& =\sigma_{\beta} p_{(\hat{\alpha}, \check{\alpha})} p_{(\hat{\beta}, \check{\beta})}-\sigma p_{(\hat{\alpha}, \hat{\beta})} p_{(\breve{\beta}, \check{\alpha})}+\text { lower order terms, }
\end{aligned}
$$

where the second equality holds by a similar computation in $\left(\begin{array}{c}\langle n\rangle \\ n\end{array}\right)_{d}$. The proof is complete.

The next result shows that the algebra with straightening law just constructed is indeed the coordinate ring of $L \mathcal{Q}_{d}(n)$.

Theorem 3.15. $\mathbb{C}\left[\left(\begin{array}{c}\langle n\rangle \\ n\end{array}\right)_{d}\right] /\left\langle I_{d, n}+L_{d, n}\right\rangle \cong \mathbb{C}\left[L \mathcal{Q}_{d}(n)\right]$.

Proof. Let $I^{\prime}:=I\left(L \mathcal{Q}_{d}(n)\right)$. Clearly, we have $I_{d, n}+L_{d, n} \subset I^{\prime}$. Since the degree and codimension of these ideals are equal, $I^{\prime}$ is nilpotent modulo $I_{d, n}+L_{d, n}$. On the other hand $I_{d, n}+L_{d, n}$ is radical, so $I_{d, n}+L_{d, n}=I^{\prime}$.

Corollary 3.16. The coordinate ring of any Schubert subvariety of $L \mathcal{Q}_{d}(n)$ is an algebra with straightening law on a doset, hence Cohen-Macaulay and Koszul.

Proof. For $\alpha^{(a)} \in \mathcal{D}_{d, n}$, the Schubert variety $X_{\alpha^{(a)}}$ is defined by the vanishing of the Plücker coordinates $p_{(\beta, \gamma)}^{(b)}$ for $\gamma^{(b)} \not \leq \alpha^{(a)}$. The four conditions in Definition 2.45 are stable upon setting these variables to zero, so we obtain an algebra with straightening law on the doset $\left\{(\beta, \gamma)^{(b)} \in \mathcal{D}_{d, n} \mid \gamma^{(b)} \leq \alpha^{(a)}\right\}$

Let $\mathcal{D} \subset \mathcal{P} \times \mathcal{P}$ be a doset on the poset $\mathcal{P}, A$ any algebra with straightening law on $\mathcal{D}$, and $\mathbb{C}\{\mathcal{P}\}$ the unique discrete algebra with straightening law on $\mathcal{P}$. That is $\mathbb{C}\{\mathcal{P}\}$ has algebra generators corresponding to the elements of $\mathcal{P}$, and the straightening 
relations are $\alpha \beta=0$ if $\alpha$ and $\beta$ are incomparable elements of $\mathcal{P}$. Then $A$ is CohenMacaulay if and only if $\mathbb{C}\{\mathcal{P}\}$ is Cohen-Macaulay [9].

On the other hand, $\mathbb{C}\{\mathcal{P}\}$ is the face ring of the order complex of $\mathcal{P}$. The order complex of a locally upper semimodular poset is shellable. The face ring of a shellable simplicial complex is Cohen-Macaulay [4]. By Proposition 2.53, any interval in the poset $\mathcal{P}_{d, n}$ is a distributive lattice, hence locally upper semimodular. This proves that $\mathbb{C}\left[L \mathcal{Q}_{d}(n)\right]$ is Cohen-Macaulay. The Koszul property is a consequence of the quadratic Gröbner basis consisting of the straightening relations.

\section{Representation-theoretic interpretation}

Let $G$ be $\operatorname{SL}(V)(\operatorname{dim} V=n+1)$ or $\operatorname{Sp}(V)(\operatorname{dim} V=2 n)$ and $\mathrm{L}(\omega)$ the irreducible representation with highest weight $\omega$, where $\omega:=\omega_{k}$ is the $k^{\text {th }}$ fundamental weight (for $G=\operatorname{Sp}(V)$, we assume that $k=n$ ). The group $\mathrm{SL}_{2}(\mathbb{C}) \times G$ acts diagonally on $S^{2}\left(S^{d} \mathbb{C}^{2} \otimes \mathrm{L}(\omega)\right)$, the degree-2 component of the homogeneous coordinate ring $S^{\bullet}\left(S^{d} \mathbb{C}^{2} \otimes V\right)$. The Drinfel'd Grassmannian $\mathcal{Q}_{d}\left(G / P_{k}\right)$ is invariant under $\mathrm{SL}_{2}(\mathbb{C}) \times G$, and so $S^{2}\left(S^{d} \mathbb{C}^{2} \otimes V\right)$ decomposes as a sum of the degree-2 components $A_{2}$ and $I_{2}$ of the coordinate ring and the defining ideal, respectively. Results in [41] and Theorem 3.14 imply that $I_{2}$ generates the full ideal $I\left(\mathcal{Q}_{d}\left(G / P_{k}\right)\right)$.

We answer the following question:

Question 3.17. How do $A_{2}$ and $I_{2}$ decompose into irreducible modules for the action of the semisimple group $\mathrm{SL}_{2}(\mathbb{C}) \times G$ ?

We use the properties of Schur modules [13, 43], which are the irreducible representations of $\mathrm{SL}(V)$. For a weight $\omega=\sum_{i=1}^{n} a_{i} \omega_{i}$ we have

$$
\mathrm{L}(\omega) \cong L_{\lambda} V
$$


where $\lambda=\left(n^{a_{n}}, \ldots, 1^{a_{1}}\right)$ and $L_{\lambda} V$ denotes the Schur module associated to $\lambda$. Our arguments apply to the case $G=\operatorname{Sp}(V)$ by virtue of Lemma 3.18 below. Suppose that the partition $\lambda=\left(\lambda_{1}, \lambda_{2}\right)$ has two rows. Recall from Chapter II that the Schur module associated to a partition $\lambda$ is defined as the image of the map

$$
\phi_{\lambda}: \wedge^{\lambda_{1}} V \otimes \wedge^{\lambda_{2}} V \rightarrow S^{\lambda_{1}^{\prime}} V \otimes \cdots \otimes S^{\lambda_{r}^{\prime}} V
$$

defined as the composition of the exterior diagonal

$$
\delta: \wedge^{\lambda_{1}} V \otimes \wedge^{\lambda_{2}} V \rightarrow \bigotimes_{i, j} V_{i, j}
$$

followed by the multiplication map

$$
m: \bigotimes_{i, j} V_{i, j} \rightarrow S^{\lambda_{1}^{\prime}} V \otimes \cdots \otimes S^{\lambda_{r}^{\prime}} V
$$

On $\bigotimes_{i, j} V_{i, j}$ we have homomorphisms $\tau_{k, \ell}: V^{\otimes N} \rightarrow V^{\otimes(N-2)}$ where $N:=|\lambda|$, given by contraction with the symplectic form $\Omega$ :

$$
\tau_{k, \ell}\left(v_{1} \otimes \cdots \otimes v_{N}\right):=\Omega\left(v_{k}, v_{\ell}\right) v_{1} \otimes \cdots \otimes \hat{v}_{k} \otimes \cdots \otimes \hat{v}_{\ell} \otimes \cdots \otimes v_{N}
$$

Lemma 3.18. Let $L_{\langle\lambda\rangle} V$ denote the irreducible representation of $\operatorname{Sp}(V)$ with highest weight $\lambda$.

1. $L_{\langle\lambda\rangle} V$ is the image in $S^{\lambda_{1}^{\prime}} V \otimes \cdots \otimes S^{\lambda_{r}^{\prime}} V$ of the intersection of $\operatorname{im}(\delta)$ with the kernels of all contraction maps $V^{\otimes N} \rightarrow V^{\otimes(N-2)}$.

2. Let $K:=\bigcap_{k=1,2}\left(\bigcap_{i, j \in\left[\lambda_{k}\right]} \operatorname{ker} \tau_{(1, i),(1, j)}\right)$ be the intersection of the kernels of contractions involving two boxes in the same row. If $i \in\left[\lambda_{1}\right], j \in\left[\lambda_{2}\right]$, and $i \neq j$, then $K \cap \operatorname{im}(\delta) \subset \operatorname{ker} \tau_{(1, i),(2, j)}+\delta\left(\operatorname{ker} \phi_{\lambda}\right)$. Thus $L_{\langle\lambda\rangle} V=\operatorname{im}(K \cap \operatorname{im}(\delta))$.

3. $L_{\langle\lambda\rangle} V$ has a basis consisting of standard tableaux of shape $\lambda$ such that each row is filled with an admissible pair of sequences. 
Proof. Statement (1) is well-known; see [13, Section 17.3]. For Statement (2), let $T \in K \cap \operatorname{im} \delta$. In particular, $T \in \operatorname{ker} \tau_{(1, i),(1, j)}$. Let $\sigma: V^{\otimes N} \rightarrow V^{\otimes N}$ be the map exchanging the $(1, j)$ - and $(2, j)$-entries. Then $m(T)=m(\sigma(T))$, and $\sigma(T) \in$ $\operatorname{ker}\left(\tau_{(1, i),(2, j)}\right)$. Thus $T-\sigma(T) \in \delta\left(\operatorname{ker} \phi_{\lambda}\right)$, hence $T \in \operatorname{ker} \tau_{(1, i),(1, j)}+\delta\left(\operatorname{ker} \phi_{\lambda}\right)$. The third statement is a consequence of the second and the combinatorics of bases of fundamental representations of $\operatorname{Sp}(V)$.

The next step is to determine how $S^{2}\left(S^{d} \mathbb{C}^{2} \otimes \mathrm{L}(\omega)\right)$ itself decomposes. Define the partition $\theta_{p}:=(k+p, k-p)$ for $p=1, \ldots, \min \{k, n-k\}$ and $\theta_{p}:=\emptyset$ for all other integer values of $p$, with the convention that $V_{\emptyset}=0$. Also, for $\operatorname{Sp}(V)$ we adopt the convention that $L_{(n+p)} V=L_{(n-p)} V$.

Proposition 3.19. The $\mathrm{SL}_{2}(\mathbb{C}) \times G$-module $S^{2}\left(S^{d} \mathbb{C}^{2} \otimes \mathrm{L}(\omega)\right)$ is isomorphic to

$$
\left(\bigoplus_{l \geq 0} \bigoplus_{m \geq 0} S^{2 d-4 l} \mathbb{C}^{2} \otimes L_{\theta_{2 m}} V\right) \oplus\left(\bigoplus_{l \geq 0} \bigoplus_{m \geq 0} S^{2 d-4 l-2} \mathbb{C}^{2} \otimes L_{\theta_{2 m+1}} V\right)
$$

Proof. We have

$$
S^{2}\left(S^{d} \mathbb{C}^{2} \otimes \mathrm{L}(\omega)\right) \cong\left(S^{2}\left(S^{d} \mathbb{C}^{2}\right) \otimes S^{2} \mathrm{~L}(\omega)\right) \oplus\left(\wedge^{2}\left(S^{d} \mathbb{C}^{2}\right) \otimes \wedge^{2} \mathrm{~L}(\omega)\right)
$$

Each module on the right decomposes further:

$$
\begin{aligned}
S^{2}\left(S^{d} \mathbb{C}^{2}\right) & \cong \bigoplus_{k \geq 0} S^{2 d-4 k} \mathbb{C}^{2} \\
\wedge^{2}\left(S^{d} \mathbb{C}^{2}\right) & \cong \bigoplus_{k \geq 0} S^{2 d-4 k-2} \mathbb{C}^{2}, \\
S^{2} \mathrm{~L}(\omega) & \cong \bigoplus_{m \geq 0} L_{\theta_{2 m}} V
\end{aligned}
$$


and

$$
\wedge^{2} \mathrm{~L}(\omega) \cong \bigoplus_{m \geq 0} L_{\theta_{2 m+1}} V
$$

The first two isomorphisms are as $\mathrm{SL}_{2}(\mathbb{C})$-modules and the last two are as $G$-modules $[13$, Exercise 15.32]. Since the action of $\mathrm{SL}_{2}(\mathbb{C}) \times G$ is diagonal, the resulting direct sum is an irreducible decomposition into $\mathrm{SL}_{2}(\mathbb{C}) \times G$-modules.

These modules can be visualized as in Figure 9. The shaded boxes correspond to the modules appearing in Proposition 3.19. We will see that the irreducible modules belonging to $A_{2}$ are those corresponding to the shaded boxes above the diagonal line (Theorem 3.22).

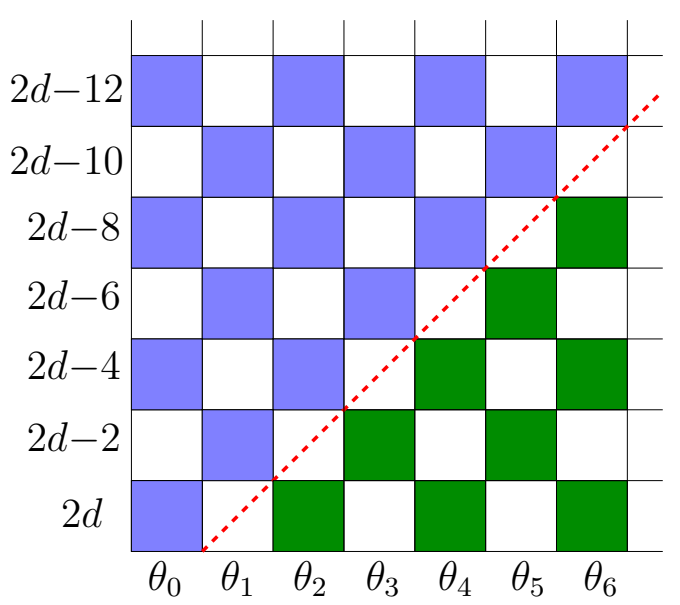

Fig. 9. The module $S^{2}\left(S^{d} \mathbb{C}^{2} \otimes \mathrm{L}(\omega)\right)$ decomposes into factors corresponding to the colored boxes. 
For a two rowed partition $\lambda$ and $p \leq \lambda_{1}-\lambda_{2}$, define $\lambda(p):=\left(\lambda_{1}+p, \lambda_{2}\right)$. If $\mu=\left(\mu_{1}, \mu_{2}\right) \subset \lambda=\left(\lambda_{1}, \lambda_{2}\right)$, define $\lambda / \mu(p):=\lambda(p) / \mu(p)$. Set $\kappa(p):=(k+p, k) /(p, 0)$. Theorem 3.14 and the results of [41] imply that $A_{2}$ is spanned by the monomials $p_{\alpha}^{(a)} p_{\beta}^{(b)}$, where the tableau obtained by filling the shape $\kappa(b-a)$ with the entries in $\alpha$ (in the first row) and $\beta$ (in the second row) is standard.

Theorem 3.20. The G-module $A_{2}$ is isomorphic to

$$
\bigoplus_{p=0}^{d}\left(L_{\kappa(p)} V\right)^{\oplus(d-p)}
$$

where $L_{\kappa(p)} V$ denotes the Schur module corresponding to the skew Young diagram $\kappa(p):=(k+p, k) /(p, 0)$.

The connection between the representations appearing in Proposition 3.19 and Theorem 3.20 is given by the following lemma [43, p. 78, Exercise 3].

Lemma 3.21. Let $\mu \subset \lambda$ be two-rowed partitions.

1. There exists a surjective $G$-morphism

$$
\psi: L_{\lambda / \mu} V \rightarrow L_{\lambda / \mu(-1)} V
$$

2. $\operatorname{ker} \psi \cong L_{\left(\lambda_{1}-\mu_{2}, \lambda_{2}-\mu_{1}\right)} V$

Proof. The module $L_{\lambda / \mu} V$ is spanned by fillings of $\lambda / \mu$ with vectors in $V$. The map $\psi$ is defined in terms of this basis by shifting the first row of a tableau one unit to the left. This map is surjective, and the kernel is the preimage of the submodule spanned by sums of the form

$$
\begin{array}{r}
\sum_{\sigma \in \mathfrak{S}_{\lambda_{1}-\mu_{2}+1}^{u+\mu_{1}}}(-1)^{\ell(\sigma)} U_{1} \wedge \cdots \wedge U_{u} \wedge W_{\sigma(1)} \wedge \cdots \wedge W_{\sigma\left(u-\mu_{1}+1\right)} \\
\otimes W_{\sigma\left(u-\mu_{1}+2\right)} \wedge \cdots \wedge W_{\sigma\left(\lambda_{1}-\mu_{2}+1\right)} \wedge V_{1} \wedge \cdots \wedge V_{v}
\end{array}
$$


where $u+v=\lambda_{2}-\mu_{1}-1$ and $U_{i}, V_{j}, W_{k}$ are elements of $V$ for $i=1, \ldots, u, j=1, \ldots, v$, $k=1, \ldots, \lambda_{1}-\mu_{2}+1$ and $\mathfrak{S}_{s}^{r}$ denotes the set of permutations $\sigma$ on $s$ letters such that $\sigma(1)<\cdots<\sigma(r)$ and $\sigma(r+1)<\cdots<\sigma(s)$. These sums over shuffles can be thought of graphically in terms of a Young scheme, in which the vectors $U_{1}, \ldots, U_{u}$ and $V_{1}, \ldots, V_{v}$ are positioned in the appropriately labeled boxes, and the vectors $W_{1}, \ldots, W_{\lambda_{1}-\mu_{2}+1}$ are shuffled (with signs) among the boxes labeled with a $\bullet$. See Figures 10 and 11.

Thus, ker $\psi$ consists of linear combinations of tableaux corresponding to the Young schemes in which the shuffled entries in the two rows just fail to overlap.

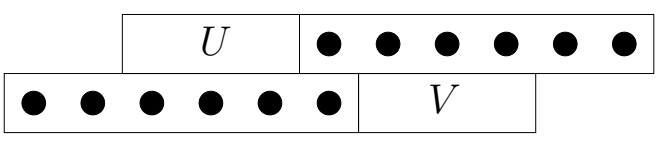

Fig. 10. A Young scheme on $\lambda / \mu(-1)$

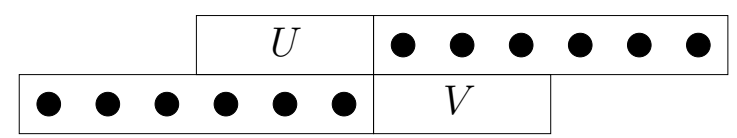

Fig. 11. A Young scheme on $\lambda / \mu$ 
Any such linear combination lies in the span of the ordinary (overlapping) Young schemes and the non-overlapping Young scheme obtained by taking $u=0$. The former vanish on $L_{\lambda / \mu(-1)} V$, and the latter is equivalent to the condition that the resulting linear combination is alternating in the vectors $U_{1}, \ldots, U_{u}, W_{1}, \ldots, W_{\lambda_{1}-\mu_{2}+1}$. Therefore we obtain a $(G$-linear) isomorphism

$$
\operatorname{ker} \psi \rightarrow L_{\left(\lambda_{1}-\mu_{2}, \lambda_{2}-\mu_{1}\right)} V
$$

by sending the sum over shuffles of a tableau on $\lambda / \mu$ to the standard tableau on $\left(\lambda_{1}-\mu_{2}, \lambda_{2}-\mu_{1}\right)$ given by putting the vectors $U_{1}, \ldots, U_{u}, W_{1}, \ldots, W_{\lambda_{1}-\mu_{2}+1}$ in order on the first row.

It follows that

$$
L_{\kappa(p)} V \cong \bigoplus_{m=0}^{p} L_{\theta_{m}} V
$$

Using Proposition 3.19, we identify which modules appear in the irreducible decomposition of $A_{2}$. This is a sum over $m$ of some $\mathrm{SL}_{2}(\mathbb{C}) \times G$-modules $M \otimes L_{\theta_{m}} V$. These come from the modules $L_{\kappa(p)} V$ for $p=m, \ldots, d$. The $\mathrm{SL}_{2}(\mathbb{C})$-weights appearing in these modules are of the form $2 d-2 p-4 q$ for $p=m, \ldots, d, q=0, \ldots, d-m$. The parameter $q$ corresponds to the $d-m+1$ copies of $L_{\kappa(p)} V$ spanned by $p_{\alpha}^{(a)} p_{\beta}^{(b)}$ (with $p=b-a \geq 0)$ such that the tableau obtained by filling the skew partition $\kappa(p)$ with the entries of $\alpha$ in the first row and $\beta$ in the second row is standard. Thus, for $p-m \equiv 0 \bmod 2$, the contributions from $L_{\kappa(p)}$ and $L_{\kappa(p+1)}$ are exactly the weights of $S^{2 d-2 m-4 p} \mathbb{C}^{2}$, so the modules $S^{2 d-2 m-4 p} \mathbb{C}^{2} \otimes L_{\theta_{m}} V$ are irreducible components of $A_{2}$. Summing over $m=0, \ldots, d$, we have:

Theorem 3.22. The irreducible decomposition of $A_{2}$ as an $\mathrm{SL}_{2}(\mathbb{C}) \times G$-module is

$$
A_{2} \cong \bigoplus_{m=0}^{d} \bigoplus_{p=0}^{\left\lfloor\frac{d-m}{2}\right\rfloor} S^{2 d-2 m-4 p} \mathbb{C}^{2} \otimes L_{\theta_{m}} V
$$


The summands of $A_{2}$ described in Theorem 3.22 are those corresponding to the boxes above the diagonal line in Figure 9. It follows that the defining ideal is generated by the direct sum of modules corresponding to the boxes below the line. 


\section{CHAPTER IV}

\section{SUMMARY}

The Grassmannian and Lagrangian Grassmannian are important test cases for understanding the flag varieties. Indeed, these were among the first spaces to which standard monomial theory was successfully applied [38, 30, 28, 29, 31]. While flag varieties provided the initial motivation, standard monomial theory has been applied to the closely related Bott-Samelson varieties, which give a resolution of singularities of Schubert varieties $[26,27,25]$. As the Drinfel'd flag varieties are singular, this suggests that one might hope to find a resolution of its singularities which also admits a straightening law.

The current situation with respect to the Drinfel'd flag varieties is similar to the early stages of the development of classical standard monomial theory. Taken together with the results of Sottile and Sturmfels [41], our results suggest that similar results might be obtained for other Drinfel'd flag varieties. The work of Littelmann [33] provided the tools needed for a fully general standard monomial theory for flag varieties (avoiding all considerations of special cases) [24]. It is interesting to ask if such an approach can be applied to the Drinfel'd flag varieties. One motivation for this would be a new geometric derivation of intersection numbers in quantum cohomology.

General results of algebras with straightening law can be found in $[3,7,8,5$, 9]. The results presented in Chapter III, Section A represent some progress in this direction, and more might yet be done. For example, any Schubert variety of the Drinfel'd Grassmannian admits a deformation to a toric variety [41], which implies that the Schubert variety is normal and has rational singularities. In some special cases, similar results exist for Schubert varieties of a flag variety $[8,15,34]$. While it is highly desirable to obtain a similar result for the Drinfel'd Lagrangian Grassmannian 
(or other Drinfel'd flag varieties), it is also interesting in general.

Question 4.1. Under what conditions does there exist a toric deformation of an algebra with straightening law?

The algebras with straightening law are a interesting class of finitely generated commutative algebras, and arise in vital areas of algebraic geometry. Answers to questions such as Question 4.1 would have important consequences; for example, that the associated varieties are normal with rational singularities. 


\section{REFERENCES}

[1] W. Borel, Linear algebraic groups, Graduate Texts in Mathematics, vol. 126, Springer-Verlag, New York, 1992.

[2] A. Braverman, Spaces of quasi-maps into the flag varieties and their applications, International Congress of Mathematicians. Vol. II, Eur. Math. Soc., Zürich, 2006, pp. $1145-1170$.

[3] W. Bruns, Additions to the theory of algebras with straightening law, Commutative Algebra, 1989, pp. 111-138.

[4] W. Bruns and J. Herzog, Cohen-Macaulay rings, Cambridge Studies in Advanced Mathematics, vol. 39, Cambridge University Press, 1993.

[5] D. Eisenbud C. De Concini and C. Procesi, Hodge algebras, vol. 91, Société Mathématique de France, 1982.

[6] L. Chen, Poincaré polynomials of hyperquot schemes, Math. Ann. 321 (2001), no. $2,235-251$.

[7] R. Chirivì, LS algebras and application to Schubert varieties, Transform. Groups 5 (2000), no. 3, 245-264.

[8] _ Deformation and Cohen-Macaulayness of the multicone over the flag variety, Comment. Math. Helv. 76 (2001), no. 3, 436-466.

[9] C. De Concini and V. Lakshmibai, Arithmetic Cohen-Macaulayness and arithmetic normality for Schubert varieties, Bull. AMS (1979), 432-435.

[10] D. Cox, J. Little, and D. O'Shea, Ideals, varieties, and algorithms, Undergraduate Texts in Mathematics, Springer-Verlag, New York, 1992. 
[11] D. Eisenbud, Commutative algebra with a view toward Algebraic Geometry, Graduate Texts in Mathematics, vol. 150, Springer-Verlag, New York, 1995.

[12] W. Fulton, Young tableaux, London Mathematical Society Student Texts, vol. 35, Cambridge University Press, Cambridge, 1997.

[13] W. Fulton and J. Harris, Representation Theory: A First Course, Graduate Texts in Mathematics, vol. 129, Springer-Verlag, New York, 1999.

[14] W. Fulton and R. Pandharipande, Notes on stable maps and quantum cohomology, Algebraic geometry — Santa Cruz 1995, Proc. Sympos. Pure Math., vol. 62, Amer. Math. Soc., Providence, RI, 1997, pp. 45-96.

[15] N. Gonciulea and V. Lakshmibai, Gröbner bases and standard monomial bases, C. R. Acad. Sci.Paris Sr. I Math. 322 (1996), 255-260.

[16] G.-M. Greuel, G. Pfister, and H. Schönemann, Singular 2.0, A Computer Algebra System for Polynomial Computations, Centre for Computer Algebra, University of Kaiserslautern, 2001, http://www.singular.uni-kl.de.

[17] J. Harris, Algebraic geometry, a first course, Graduate Texts in Mathematics, vol. 133, Springer-Verlag, New York, 1992.

[18] R. Hartshorne, Algebraic geometry, Springer-Verlag, New York, 1977, Graduate Texts in Mathematics, No. 52.

[19] W.V.D. Hodge, Some enumerative results in the theory of forms, Proc. Camb. Phil. Soc. 39 (1943), 22-30.

[20] S. Kleiman, Chasles's enumerative theory of conics: a historical introduction, Studies in algebraic geometry, MAA Stud. Math., vol. 20, Math. Assoc. America, Washington, D.C., 1980, pp. 117-138. 
[21] M. Kontsevich, Enumeration of rational curves via torus actions, The moduli space of curves (Texel Island, 1994), Progr. Math., vol. 129, Birkhäuser Boston, Boston, MA, 1995, pp. 335-368.

[22] A. Kuznetsov, Laumon's resolution of Drinfeld's compactification is small, Math. Res. Lett. 4 (1997), 349-364.

[23] V. Lakshmibai, The development of standard monomial theory - II, A Tribute to C. S. Seshadri, Hindustan Book Agency, 2003, pp. 283-309.

[24] V. Lakshmibai, P. Littelmann, and P. Magyar, Standard monomial theory and applications, Representation theories and algebraic geometry (Montreal, PQ, 1997), NATO Adv. Sci. Inst. Ser. C Math. Phys. Sci., vol. 514, Kluwer Acad. Publ., Dordrecht, 1998, Notes by Rupert W. T. Yu, pp. 319-364.

[25] _ Standard monomial theory for Bott-Samelson varieties, Compositio Math. 130 (2002), no. 3, 293-318.

[26] V. Lakshmibai and P. Magyar, Standard monomial theory for Bott-Samelson varieties, C. R. Acad. Sci. Paris Sér. I Math. 324 (1997), no. 11, 1211-1215.

[27]_ Standard monomial theory for Bott-Samelson varieties of GL(n), Publ. Res. Inst. Math. Sci. 34 (1998), no. 3, 229-248.

[28] V. Lakshmibai, C. Musili, and C. S. Seshadri, Geometry of G/P. III. Standard monomial theory for a quasi-minuscule P, Proc. Indian Acad. Sci. Sect. A Math. Sci. 88 (1979), no. 3, 93-177. MR MR561813 (81g:14023c)

[29] _ Geometry of G/P. IV. Standard monomial theory for classical types, Proc. Indian Acad. Sci. Sect. A Math. Sci. 88 (1979), no. 4, 279-362. MR $\operatorname{MR553746~(81g:14023d)~}$ 
[30] V. Lakshmibai and C. S. Seshadri, Geometry of G/P. II. The work of de Concini and Procesi and the basic conjectures, Proc. Indian Acad. Sci. Sect. A 87 (1978), no. $2,1-54$.

[31] _ Geometry of G/P. V, J. Algebra 100 (1986), no. 2, 462-557.

[32] G. Laumon, Faisceaux automorphes liés aux séries d'Eisenstein, Automorphic forms, Shimura varieties, and $L$-functions, Vol. I (Ann Arbor, MI, 1988), Perspect. Math., vol. 10, Academic Press, Boston, MA, 1990, pp. 227-281.

[33] P. Littelmann, Contracting modules and standard monomial theory for symmetrizable Kac-Moody algebras, J. Amer. Math. Soc. 11 (1998), no. 3, 551-567.

[34] E. Miller and B. Sturmfels, Combinatorial commutative algebra, Graduate Texts in Mathematics, vol. 227, Springer-Verlag, New York, 2005.

[35] C. Musili, The development of standard monomial theory - I, A Tribute to C. S. Seshadri, Hindustan Book Agency, 2003, pp. 385-420.

[36] J. Rosenthal, On dynamic feedback compensation and compactification of systems, SIAM J. Control Optim. 32 (1994), no. 1, 279-296.

[37] H. Schubert, Beziehungen zwischen den linearen Räumen auferlegbaren charakteristischen Bedingungen, Math. Ann. 38 (1891), no. 4, 598-602.

[38] C. S. Seshadri, Geometry of G/P. I. Theory of standard monomials for minuscule representations, C. P. Ramanujam - a tribute, Tata Inst. Fund. Res. Studies in Math., vol. 8, Springer, Berlin, 1978, pp. 207-239.

[39] F. Sottile, Real rational curves in Grassmannians, J. Amer. Math. Soc. 13 (2000), no. $2,333-341$. 
[40] _ Rational curves on Grassmannians: systems theory, reality, and transversality, Advances in algebraic geometry motivated by physics (Lowell, MA, 2000), Contemp. Math., vol. 276, Amer. Math. Soc., Providence, RI, 2001, pp. 9-42.

[41] F. Sottile and B. Sturmfels, A sagbi basis for the quantum Grassmannian, J. Pure and Appl. Alg. 158 (2001), 347-366.

[42] S. A. Strømme, On parametrized rational curves in Grassmann varieties, Space curves (Rocca di Papa, 1985), Lecture Notes in Math., vol. 1266, Springer, Berlin, 1987, pp. 251-272.

[43] J. Weyman, Cohomology of vector bundles and syzygies, Cambridge Tracts in Mathematics, vol. 149, Cambridge University Press, Cambridge, 2003. 


\section{VITA}

Name: $\quad$ James Vincent Ruffo

Address: $\quad$ Department of Mathematics

Mailstop 3368

Texas A\&M University

College Station, TX 77840

Email Address: jruffo@math.tamu.edu

Education: $\quad$ B.A., Mathematics, University of Rochester, 1996

B.S., Physics, University of Rochester, 1996

M.A., Mathematics, University of Massachusetts-Amherst, 2004 